TRANSACTIONS OF THE

AMERICAN MATHEMATICAL SOCIETY

Volume 351, Number 5 , Pages 1919-1945

S 0002-9947(99)02110-8

Article electronically published on January 26, 1999

\title{
ON THE NUMBER OF RADIALLY SYMMETRIC SOLUTIONS TO DIRICHLET PROBLEMS WITH JUMPING NONLINEARITIES OF SUPERLINEAR ORDER
}

\author{
ALFONSO CASTRO AND HENDRIK J. KUIPER
}

\begin{abstract}
This paper is concerned with the multiplicity of radially symmetric solutions $u(x)$ to the Dirichlet problem$$
\Delta u+f(u)=h(x)+c \phi(x)
$$

on the unit ball $\Omega \subset \mathbf{R}^{N}$ with boundary condition $u=0$ on $\partial \Omega$. Here $\phi(x)$ is a positive function and $f(u)$ is a function that is superlinear (but of subcritical growth) for large positive $u$, while for large negative $u$ we have that $f^{\prime}(u)<\mu$, where $\mu$ is the smallest positive eigenvalue for $\Delta \psi+\mu \psi=0$ in $\Omega$ with $\psi=0$ on $\partial \Omega$. It is shown that, given any integer $k \geq 0$, the value $c$ may be chosen so large that there are $2 k+1$ solutions with $k$ or less interior nodes. Existence of positive solutions is excluded for large enough values of $c$.
\end{abstract}

\section{INTRODUCTION}

Let $\Delta$ denote the Laplacian operator on $\Omega \subset R^{N}$ with Dirichlet boundary conditions (i.e., $\operatorname{Dom}(\Delta)=H_{0}^{1}(\Omega) \subset L^{2}(\Omega)$ ), and consider the semilinear Dirichlet problem

$$
\Delta u+f(u)=h(x)+c \phi_{1}(x) \text { in } \Omega,
$$

where $f^{\prime}(-\infty):=\lim _{u \rightarrow-\infty} f^{\prime}(u)<f^{\prime}(\infty):=\lim _{u \rightarrow \infty} f^{\prime}(u)$ and $\phi_{1}$ is a positive function. Motivated by the well-known result of Ambrosetti and Prodi [1], there has been a great deal of work devoted to understanding the relationship between the number of solutions to this problem and the number of eigenvalues of $-\Delta$ that lie in the interval $\left(f^{\prime}(-\infty), f^{\prime}(\infty)\right)$. A history of the problem as well as a substantial bibliography may be found in the review article by Lazer and McKenna [5]. In that article the authors pose several open problems. One of these is whether the problem with $f^{\prime}(-\infty)<\lambda_{1}<f^{\prime}(\infty)=\infty, \lambda_{1}$ the principal eigenvalue of $-\Delta$, can have arbitrarily many solutions provided $c$ is chosen sufficiently high. This question was partially answered in [3], where the authors investigated the problem on the unit ball with $h(x)=h(|x|)$. It was shown that there do indeed exist arbitrarily many (radially symmetric) solutions provided $c$ is chosen sufficiently high. The solutions that were found are negative at the origin. In the present paper we will show that there exist two such arbitrarily large families of solutions, the other consisting of solutions that are positive at the origin. The existence of two such

Received by the editors April 24, 1996.

1991 Mathematics Subject Classification. Primary 35J65, 34A10.

Key words and phrases. Radially symmetric, Dirichlet problem, superlinear jumping nonlinearity, nodal curves, critical exponent.

(C)1999 American Mathematical Society 
families of solutions in case $-\infty<f^{\prime}(-\infty)<f^{\prime}(\infty)<\infty$ was shown in [2]. Our result applies to somewhat more general situations than those treated in [3], and the proof is based on the study of asymptotic behavior, on estimates gathered through the use of two energy functions, and the shooting method. The solutions with $u(0)<0$ are obtained by shooting solutions from $|x|=1$, while those with $u(0)>0$ are obtained by matching solutions obtained by shooting from $|x|=1$ with solutions obtained by shooting from the origin.

\section{The Main Result}

Let $\mu_{N}$ be the principal eigenvalue for

$$
\begin{aligned}
-\Delta \psi(x) & =\mu \psi(x), \quad x \in R^{N}, \quad|x|<1, \\
\psi(x) & =0 \quad \text { if } r \equiv|x|=1,
\end{aligned}
$$

where $N \geq 2$. It is not difficult to see that $r^{1-N / 2} J_{N / 2-1}\left(\sqrt{\mu_{N}} r\right)$ is a principal eigenfunction, so that $\sqrt{\mu_{N}}$ is the location of the first positive root of $J_{N / 2-1}$. We will be concerned with the radially symmetric solutions to the Dirichlet problem

$$
\begin{gathered}
-\Delta u(x)=\tilde{f}(u(x))-C \tilde{\phi}(|x|)+\tilde{\psi}(|x|, u(x)), \quad x \in R^{N}, \quad|x|<1, \\
u(x)=0 \quad \text { if }|x|=1,
\end{gathered}
$$

where $C$ is a positive constant, $\tilde{f}$ is superlinear at $\infty$ while $\lim \sup _{u \rightarrow-\infty} \tilde{f}(u)<\mu_{N}$. It will be shown that by choosing $C$ sufficiently large we can ensure the existence of an arbitrarily large number of solutions with prescribed nodal properties (with the possible exception of positive solutions). We impose the following restrictions:

1. $\tilde{H}_{1} . \tilde{\phi}, \tilde{\psi}$ and $\tilde{\psi}_{u}$ are bounded and continuous, $\tilde{\phi}(r)>0$ on $[0,1]$, and

$$
\lim _{u \rightarrow-\infty} \tilde{\psi}_{u}=0 .
$$

2. $\tilde{H}_{2} . \tilde{f} \in C^{1}(R)$ and $\lim _{u \rightarrow \infty} \tilde{f}^{\prime}(u)=\infty$.

3. $\tilde{H}_{3}$. If $N=2$ we assume

$$
\liminf _{s \rightarrow \infty} \int_{0}^{s} \tilde{f}(\sigma) d \sigma /(s \tilde{f}(s))>0 .
$$

4. If $N>2$ we assume $\frac{2 N}{N-2} \underline{G}(\tau) \geq \bar{g}(\tau)$ on $0<\tau \leq 1$ with strict inequality on a set of positive measure, where

$$
\underline{G}(\tau)=\int_{0}^{\tau} \underline{g}(\sigma) d \sigma, \quad \underline{g}(\tau)=\liminf _{M \rightarrow \infty} \tilde{f}(\tau M) / \tilde{f}(M)
$$

and

$$
\bar{g}(\tau)=\limsup _{M \rightarrow \infty} \tilde{f}(\tau M) / \tilde{f}(M) .
$$

5. $\tilde{H}_{4} \cdot \lim \sup _{u \rightarrow-\infty} \tilde{f}^{\prime}(u)<\mu_{N}$ and $\lim \sup _{u \rightarrow-\infty} f(u)<\infty$.

Our main result is:

Theorem. Suppose $\tilde{H}_{1}-\tilde{H}_{4}$ are satisfied and $K$ is any nonnegative integer. There exists a constant $C_{K}>0$ such that whenever $C>C_{K}$ then

(i) For each integer $0 \leq k \leq K$ problem $(D E)+(B C)$ has a radially symmetric solution $u_{k}^{(-)}$with $u_{k}^{(-)}(0)<0$ and such that $u_{k}^{(-)}$has precisely $k$ nodal surfaces in $\Omega:=\left\{x \in R^{N}:|x|<1\right\}$. 
(ii) For each integer $1 \leq k \leq K$ problem $(D E)+(B C)$ has a radially symmetric solution $u_{k}^{(+)}$with $u_{k}^{(+)}(0)>0$ and such that $u_{k}^{(+)}$has $k$ nodal surfaces in $\Omega$.

(iii) Problem $(D E)+(B C)$ has no positive solution if $C$ is sufficiently high.

\section{Other Forms of the Problem}

It is easy to see that we may write $\tilde{f}(u)=C_{f}+f(u)+f_{0}(u)$, where $C_{f}$ is any constant greater than $\lim \sup _{u \rightarrow-\infty} \tilde{f}(u), f_{0}$ is a $C^{1}$ function of compact support, and $f$ is a $C^{1}$ function satisfying $f(0)=0, f(u) u>0$ for all $u \neq 0, f^{\prime}(u)>0$ for $u>0, f^{\prime}(u)<\mu_{N}$ for all $u<0$. Let $\varphi(r):=\tilde{\varphi}(r) /\|\tilde{\varphi}\|$, where $\|\quad\|$ denotes the sup norm on $[0,1]$. Also let $d=\min _{0 \leq r \leq 1} \varphi(r)$ and define $\psi(r, n)=\tilde{\psi}(r, n)+f_{0}(u)+$ $C_{f}+C_{0} \tilde{\varphi}(r)$, where $C_{0}$ is chosen large enough so that $\psi(r, u) \geq 0$. Finally, let us choose $C$ large enough so that $C+C_{0}=f(B)$ for some $B \geq 0$. Then (BC) $+(\mathrm{DE})$ may be rewritten as

$$
\begin{gathered}
u^{\prime \prime}(r)=-\frac{n u^{\prime}}{r}+f(B) \varphi(r)-\psi(r, u)-f(u), \\
u^{\prime}(0)=0, \quad u(1)=0,
\end{gathered}
$$

where $n=N-1$, and hypotheses $\tilde{H}_{1}-\tilde{H}_{4}$ may be rewritten as

$H_{1}: \varphi$ is continuous on $[0,1]$, and $0<d \leq \varphi(r) \leq 1$ for all $r \in[0,1]$, where $d$ is a constant. $\psi$ and $\psi_{u}$ are bounded continuous on $[0,1] \times R, \psi(r, u) \geq 0$, and $\lim _{u \rightarrow-\infty} \psi_{u}(r, u)=0$.

$H_{2}: f \in C^{1}(R), f(0)=0, f(u) \geq 0$ and $f^{\prime}(u)>0$ for all $u \geq 0$, and

$$
\lim _{u \rightarrow \infty} f^{\prime}(u)=\infty \text {. }
$$

$H_{3}$ : If $N=2$ we assume $\liminf _{s \rightarrow \infty} \int_{0}^{s} f(\sigma) d \sigma /(s f(s))>0$. If $N>2, \bar{g}(\tau):=$ $\lim \sup _{M \rightarrow \infty} f(\tau M) / f(M), \underline{g}(\tau):=\liminf _{M \rightarrow \infty} f(\tau M) / f(M)$, and $\underline{G}(\tau):=$ $\int_{0}^{\tau} \underline{g}(\sigma) d \sigma$, then

$$
\frac{2 n+2}{n-1} \underline{G}(\tau) \geq \bar{g}(\tau) \tau \quad \text { on }(0,1] .
$$

with strict inequality on a set of positive measure.

$H_{4}$ : For $u \leq 0$ we have $f(u) \leq 0$ and $f^{\prime}(u)<\mu_{N}$.

Remark 1. $\bar{g}$ and $\underline{g}$ must be bounded and nondecreasing on $(0,1]$. If, for example, $f(u) / u^{p} \rightarrow c s t$ as $u \rightarrow \infty$ then $\underline{g}(\tau) \equiv \bar{g}(\tau)=\tau^{p}$. Here, and in the future, we use cst to denote a generic positive constant.

On occasion it will be convenient to change variables and map $(0,1]$ onto $[1, \infty)$ and transform (1) into an equation that is somewhat similar to an Emden-Fowler equation. If $n>1$ we set $s=r^{1-n}$ and $U(s)=u(r)$, so that (1) becomes

$$
U^{\prime \prime}(s)=(n-1)^{-2} s^{-2 n /(n-1)}\left[f(B) \phi\left(s^{-1 /(n-1)}\right)-\psi\left(s^{-1 /(n-1)}, U(s)\right)-f(U(s)) .\right.
$$

If $n=1$ we set $s=-\ln r$ and $U(s)=u(r)$, and (1) becomes

$$
U^{\prime \prime}(s)=e^{-2 s}\left[f(B) \phi\left(e^{-s}\right)-\psi\left(e^{-s}, U(s)\right)-f(U(s))\right] .
$$

By integrating (1) twice we may also transform it into an integral equation:

$$
u(r)=u(0)-\frac{1}{n-1} \int_{0}^{r} \sigma\left[1-(\sigma / r)^{n-1}\right][f(u(r))-f(B) \phi(\sigma)+\psi(\sigma, u(\sigma))] d \sigma
$$


if $n \geq 2$

$$
u(r)=u(0)-\int_{0}^{r} \sigma \ln (r / \sigma)[f(u(\sigma))-f(B) \varphi(\sigma)-\psi(\sigma, u(\sigma))] d \sigma .
$$

if $n=1$, or

Definitions. We define $F(u):=\int_{0}^{u} f(\sigma) d \sigma, G_{B k}(u):=F(u)-k f(B) u, k_{B}=$ $F(B) /(B f(B)), \mathcal{E}(r) \equiv \mathcal{E}_{u}(r):=\frac{1}{2} u^{\prime}(r)^{2}+F(u(r))$ and $E(r) \equiv E_{u}(r):=\mathcal{E}(r)-$ $f(B) u(r)$.

Lemma 1. (i) If $N>2$, then $H_{3}$ implies that for each $0<\epsilon<1$ there is a number $c_{\epsilon}>0$ such that

$$
(1-\epsilon) \sigma f(\sigma) \leq \frac{2 n+2}{n-1} F(\sigma) \quad \forall \sigma>c_{\epsilon}
$$

(ii) $\underline{g}$ is positive on $[0,1]$, and for each $0<\delta<1$ and each $0<\epsilon<\underline{g}(\delta)$ there exists a constant $M_{\epsilon}>0$ such that $f(\delta M) / f(M)>\underline{g}(\delta)-\epsilon$ whenever $M \geq M_{\epsilon}$.

Proof. Set $\tau=1$; then

$$
\begin{aligned}
1= & f(M) / f(M)=\bar{g}(1)<\frac{2 n+2}{n-1} \int_{0}^{1} \liminf _{W \rightarrow \infty} f\left(\tau^{\prime} W\right) / f(W) d \tau^{\prime} \\
& (\text { by Fatou's lemma }) \leq \frac{2 n+2}{n-1} \liminf _{W \rightarrow \infty} F(W) /(W f(W)) .
\end{aligned}
$$

Therefore, for sufficiently large $W$ we have

$$
1 \leq \epsilon+\frac{2 n+2}{n-1} F(W) /(W f(W)) .
$$

It is obvious that $\bar{g}$ and $\underline{g}$ are nondecreasing functions with $\bar{g}(1)=\underline{g}(1)=1$ and $\bar{g}(0)=\underline{g}(0)=0$. Also, one readily verifies that the strict inequality in $H_{3}$ implies that both $\underline{g}$ and $\bar{g}$ are positive on $[0,1]$. Now (ii) follows since

$$
\liminf _{M \rightarrow \infty} f(\delta M) / f(M)=\underline{g}(\delta)>0 .
$$

Remark 2. In case $N=2(n=1), H_{3}$ obviously implies that there is a positive number $\delta_{1}<1$ such that $\delta_{1} \sigma f(\sigma) \leq F(\sigma)$, for all sufficiently large $\sigma$. Hence,

Definition. For each $n \geq 1$ let $0<\delta_{n}<1$ and $c_{n} \geq 0$ be numbers such that

$$
\delta_{n} \sigma f(\sigma) \leq F(\sigma) \quad \forall \sigma \geq c_{n} .
$$

Definition. We will use $\mathcal{F}$ to denote the function $\mathcal{F}:[0, \infty) \rightarrow[0, \infty)$ defined by $\mathcal{F}(u)=\min \left\{f^{\prime}(v) ; v \geq u\right\}$.

Lemma 2. (i) There exists a value $s_{0}>0$ such that $f^{\prime}(s)>\max \left|\psi_{u}\right|$ for all $s \geq s_{0}$ and $f(s) \leq f\left(s_{0}\right)$ for all $s \leq s_{0}$.

(ii) There exist arbitrarily large values $B$ such that $f^{\prime}(s) \leq f^{\prime}(B)$ and $f(s)<$ $f(B)$ for all $s \leq B$.

(iii) $s^{-1} F(s)<f(s)$ for all $s \geq s_{0}, f(B)-B^{-1} F(B) \geq(B / 2) \mathcal{F}(B / 2)$ and hence $\lim _{B \rightarrow \infty} f(B)-B^{-1} F(B)=\infty$.

(iv) $G_{B k}<0$ on $(0, B)$ provided $B \geq s_{0}$ and $k>k_{B}$. 
(v) $G_{B k}$ restricted to $\left[f^{-1}(k f(B)), \infty\right)$ is increasing and has an inverse, $G_{B k}^{-1}$. $G_{B k}^{-1}(\sigma)$ is well defined for all $\sigma \geq 0$. Let $\kappa_{0}$ be a positive number such that $f^{\prime}(u) \geq$ $f^{\prime}\left(\kappa_{0}\right)$ for all $u \geq \kappa_{0}$ and such that $f\left(\kappa_{0}\right)<k f(B)$. Then

$$
G_{B k}^{-1}(\sigma) \leq 2 \kappa_{0}+3 k f(B) / f^{\prime}\left(\kappa_{0}\right)+\sqrt{2 \sigma / f^{\prime}\left(\kappa_{0}\right)}
$$

(vi) There exist constants $\lambda_{n}>[\sqrt{2}-1]^{-1}$ and $B_{n}(K)$ such that for $1 \leq \eta \leq K$

$$
\lambda_{n} \delta_{n} f(\eta B) / f(B)>\eta \quad \forall B>B_{n}(K) .
$$

The condition $\lambda_{n}>[\sqrt{2}-1]^{-1}$ is imposed merely for later convenience.

(vii) If $k \leq 1, c^{2} \geq 2$, then

$$
G_{B k}^{-1}\left(\frac{1}{2} k c^{2} B f(B)\right) \leq\left[1+1 / \lambda_{n}\right] c^{2} B .
$$

Proof. (i) and (ii) are immediate consequences of $H_{2}$, while for $s \geq s_{0}$ (iii) follows from

$$
F(s)-f(s) s=s[F(s) / s-f(s)]=s \times\{\text { average of } f \text { on }[0, s]-f(s)\} \leq 0
$$

and

$$
\begin{aligned}
f(B)-B^{-1} F(B) & =\frac{(B / 2) f(B / 2)-F(B / 2)}{B}+\frac{1}{B} \int_{B / 2}^{B} f^{\prime}(s) s d s \\
& \geq \frac{1}{2} \times\left\{\text { average of } f^{\prime}(s) \text { on }[B / 2, B]\right\} \\
& \geq(B / 2) \mathcal{F}(B / 2) \rightarrow \infty \text { as } B \rightarrow \infty
\end{aligned}
$$

(iv) follows from

$$
\begin{aligned}
G_{B k}(s) / s & =F(s) / s-k f(B)=\text { average value of } f \text { on }[0, s]-k f(B) \\
& \leq \text { average value of } f \text { on }[0, B]-k f(B) \\
& =F(B) / B-k f(B)<F(B) / B-k_{B} f(B)=0 .
\end{aligned}
$$

Note that $G_{B k}^{\prime \prime}(u)=f^{\prime}(u)>0$ on $[0, \infty)$ and $G_{B k}$ attains its (nonpositive) minimum at $f^{-1}(k f(B))$. Hence $G_{B k}^{-1}$ is well defined on $[0, \infty)$. We also see that

$$
\begin{aligned}
\sigma:=G_{B k}(u) & \geq G_{B k}\left(\kappa_{0}\right)+G_{B k}^{\prime}\left(\kappa_{0}\right)\left(u-\kappa_{0}\right)+\frac{1}{2} G_{B k}^{\prime \prime}\left(\kappa_{0}\right)\left(u-\kappa_{0}\right)^{2} \\
& \geq G_{B k}\left(\kappa_{0}\right)+\left(f\left(\kappa_{0}\right)-k f(B)\right)\left(u-\kappa_{0}\right)+\frac{1}{2} f^{\prime}\left(\kappa_{0}\right)\left(u-\kappa_{0}\right)^{2} .
\end{aligned}
$$

This implies that

or

$$
\left[\left(\frac{f\left(\kappa_{0}\right)-f(B)}{f^{\prime}\left(\kappa_{0}\right)}\right)^{2}+\frac{2\left(\sigma-G_{B k}\left(\kappa_{0}\right)\right)}{f^{\prime}\left(\kappa_{0}\right)}\right]^{1 / 2} \geq\left|u-\kappa_{0}+\frac{f\left(\kappa_{0}\right)-k f(B)}{f^{\prime}\left(\kappa_{0}\right)}\right|
$$

$$
\begin{aligned}
u & \leq \kappa_{0}-\frac{f\left(\kappa_{0}\right)-k f(B)}{f^{\prime}\left(\kappa_{0}\right)}+\left[\left(\frac{f\left(\kappa_{0}\right)-k f(B)}{f^{\prime}\left(\kappa_{0}\right)}\right)^{2}-\frac{2 G_{B k}\left(\kappa_{0}\right)}{f^{\prime}\left(\kappa_{0}\right)}\right]^{1 / 2}+\sqrt{2 \sigma / f^{\prime}\left(\kappa_{0}\right)} \\
& \leq \kappa_{0}+\frac{2\left(k f(B)-f\left(\kappa_{0}\right)\right)}{f^{\prime}\left(\kappa_{0}\right)}+\left[\frac{2\left(k f(B) \kappa_{0}-F\left(\kappa_{0}\right)\right)}{f^{\prime}\left(\kappa_{0}\right)}\right]^{1 / 2}+\sqrt{2 \sigma / f^{\prime}\left(\kappa_{0}\right)} \\
& \leq \kappa_{0}+2 k f(B) / f^{\prime}\left(\kappa_{0}\right)+\sqrt{2 k f(B) \kappa_{0} / f^{\prime}\left(\kappa_{0}\right)}+\sqrt{2 \sigma / f^{\prime}\left(\kappa_{0}\right)} \\
& \leq 2 \kappa_{0}+3 k f(B) / f^{\prime}\left(\kappa_{0}\right)+\sqrt{2 \sigma / f^{\prime}\left(\kappa_{0}\right)} .
\end{aligned}
$$


In order to prove (vi) for $n>1$ we first show that $g(\tau)=O(\tau)$ near $\tau=0$. Let $h(s):=f(s) / s$ for $s>0$. One easily sees that $h(s) \rightarrow \infty$ as $s \rightarrow \infty$. Choose a sequence $\left\{M_{i}\right\}_{i=1}^{\infty}$ such that $h\left(M_{i}\right)=\max \left\{h(s): 0 \leq s \leq M_{i}\right\}$; then

$$
f\left(\tau M_{i}\right) / f\left(M_{i}\right)=\tau M_{i} h\left(\tau M_{i}\right) /\left[M_{i} h\left(M_{i}\right)\right] \leq \tau
$$

and hence $\underline{g}(\tau) \leq \tau$. It follows that $\underline{G}(\tau) \leq \frac{1}{2} \tau^{2}$ and $\bar{g}(\tau) \leq(n+1) \tau /(n-1)$. There is a $B_{\eta}>0$ such that

$$
f(\eta B) / f(B) \geq \frac{1}{2} f(\eta B) /\left[\bar{g}\left(\eta^{-1}\right) f(\eta B)\right] \geq \frac{n-1}{2 n+2} \eta \quad \forall B \geq B_{\eta} .
$$

However, since the functions $f(\tau M) / f(M)$ are monotone in $\tau$ on compact subintervals of $(0,1]$ and for $M$ sufficiently large, the convergence $\sup _{N \geq M}\{f(\tau N) / f(N)\} \rightarrow$ $\bar{g}(\tau)$ as $N \rightarrow \infty$ is uniform. Hence the above inequality can be made to hold simultaneously for all $\eta \geq 1$ in compact sets. If $n=1$, then

$$
\begin{aligned}
f(\eta B) / f(B) & =\left[\int_{0}^{\eta B} f^{\prime}(\sigma) d \sigma /(\eta B f(\eta B))\right][\eta B f(\eta B) / f(B)] \\
& \geq \frac{1}{2} \liminf _{s \rightarrow \infty}\left\{\int_{0}^{s} f^{\prime}(\sigma) d \sigma /(s f(s)\},\right.
\end{aligned}
$$

so we may pick $B_{1}(K)=B_{1}>0$ arbitrarily and choose $\lambda_{1}$ so that the liminf in the above inequality exceeds $2 /\left(B_{1} \lambda_{1} \eta_{1}\right)$. To prove (vii) we observe that

$$
\frac{1}{2} c^{2} B f(B)\left(\lambda_{n}+2\right) \leq \frac{\left(\lambda_{n}+1\right)^{2}}{4 \lambda_{n}} c^{4} B f(B)
$$

Using (vi) with $\eta=\left(\lambda_{n}+1\right) c^{2} / 2>1$ :

$$
\begin{aligned}
\frac{1}{2} k c^{2} B f(B)\left[1+k\left(\lambda_{n}+1\right)\right] & \leq \frac{1}{2}\left(1+1 / \lambda_{n}\right) c^{2} B \lambda_{n} \delta_{n} f\left(\frac{1}{2}\left(\lambda_{n}+1\right) c^{2} B\right) \\
& \leq F\left(\frac{1}{2}\left(\lambda_{n}+1\right) c^{2} B\right) .
\end{aligned}
$$

Rearranging, we get

$$
\frac{1}{2} k c^{2} B f(B) \leq G_{B k}\left(\frac{1}{2}\left(\lambda_{n}+1\right) c^{2} B\right) .
$$

Since $\left(\lambda_{n}+1\right) c^{2} B / 2>B$, we may apply the monotone increasing function $G_{B k}^{-1}$.

Note. In what follows we will always assume $B>s_{0}$. Indeed, we will choose $B$ so large that

$$
B_{*}:=f^{-1}[f(B) d-\|\psi\|] \geq s_{0} .
$$

Lemma 3. $E^{\prime}(s) \leq 0$ whenever $u^{\prime}(s) \geq 0$, and $\mathcal{E}^{\prime}(s) \leq 0$ whenever $u^{\prime}(s) \leq 0$.

Proof. We have

$$
\begin{aligned}
E^{\prime}(s)= & u^{\prime}(s) u^{\prime \prime}(s)+f(u(s)) u^{\prime}(s)-f(B) u^{\prime}(s) \\
& -\frac{n}{r} u^{\prime}(s)^{2}+(f(B) \varphi(s)-\psi(s, u)) u^{\prime}(s)-f(B) u^{\prime}(s) \leq 0
\end{aligned}
$$


since $\|\varphi\| \leq 1$ and $\psi \geq 0$. Also,

$$
\begin{aligned}
\mathcal{E}^{\prime}(s) & =u^{\prime}(s) u^{\prime \prime}(s)+f\left(u(s) u^{\prime}(s)\right) \\
& =-\frac{n}{r} u^{\prime}(s)^{2}+[f(B) \varphi(s)-\psi(s, u)] u^{\prime}(s) \\
& \leq f\left(B_{*}\right) u^{\prime}(s) \leq 0 .
\end{aligned}
$$

\section{A. Estimates on intervals Where the SOlution is POSitive}

Suppose that $u$ satisfies equation $(1)$ on $(0,1)$ and that $u>0$ on $(\alpha, \beta)$ with $u(\alpha)=u(\beta)=0$. Let us denote $u^{\prime}(\beta)=-J$. By applying the contraction mapping principle to the map

$$
w \longmapsto f^{-1}(f(B) \phi-\psi(\cdot, w))
$$

and using the fact that $B>s_{0}$ and $f(B) \phi(r)-\psi(r, u) \geq f\left(s_{0}\right)$, one readily verifies that there is a unique function $u_{B} \in C[0,1]$ such that

$$
f\left(u_{B}(r)\right)=f(B) \phi(r)-\psi\left(r, u_{B}(r)\right), \quad 0 \leq r \leq 1,
$$

and

$$
B_{*} \leq u_{B}(r) \leq B .
$$

Lemma 4. Suppose $u$ is a solution of $(1)$ on $(0,1), u>0$ on $(\alpha, \beta) \subset(0,1)$ and $u>B$ on $\left(\alpha_{0}, \beta_{0}\right) \subset(\alpha, \beta)$. Then

$$
\beta_{0}-\alpha_{0}<\frac{2^{(n-1) / 2}}{\sqrt{\mathcal{F}(B)}} \max \left[\pi, \sqrt{\mu_{N}}\right]
$$

Proof. Let $w=u-B$; then

$$
w^{\prime \prime}+\frac{n w^{\prime}}{r}=f(B) \phi-f(B+w)-\psi(r, B+w)
$$

or

$$
\left(-r^{n} w^{\prime}\right)^{\prime}=r^{n}[f(B+w)-f(B)]+r^{n}[f(B)-f(B) \phi+\psi(r, B+w)],
$$

where the last term in the brackets is nonnegative. We may write $f(B+w(r))-$ $f(B)=f^{\prime}(\eta(r)) w(r) \geq \mathcal{F}(\eta(r)) w(r)$ for some function $\eta(r) \geq B$ and consider the problem

$$
-\left(r^{n} v^{\prime}\right)^{\prime}=r^{n} \mathcal{F}(\eta) v, \quad v\left(\alpha_{0}\right)=0, \quad v^{\prime}\left(\alpha_{0}\right)=w^{\prime}\left(\alpha_{0}\right) .
$$

By the Sturm comparison theorem [4], $v$ will achieve its smallest root greater than $\alpha_{0}$ at a point $\beta_{1}>\beta_{0}$. (The proof in [4] extends easily to the case where $w$ satisfies a differential inequality $-\left(r^{n} w^{1}\right)^{\prime} \geq r^{n} \mathcal{F}(\eta) w$ rather than an equality). Let $\sigma=\left(r-\alpha_{0}\right) /\left(\beta_{1}-\alpha_{0}\right), v(r)=V(\sigma), \eta(r)=H(\sigma)$; then $V$ is a principal eigenfunction for

$$
\begin{gathered}
-\frac{d}{d \sigma}\left\{\left[\alpha_{0}+\left(\beta_{1}-\alpha_{0}\right) \sigma\right]^{n} \frac{d V}{d v}\right\}=\lambda\left[\alpha_{0}+\left(\beta_{1}-\alpha_{0}\right) \sigma\right]^{n} \mathcal{F}(H(\sigma)) V, \\
V(0)=V(1)=0
\end{gathered}
$$


with principal eigenvalue $\lambda=\left(\beta_{1}-\alpha_{0}\right)^{2}$. Using the variational characterization of this eigenvalue, we have

$$
\begin{aligned}
\left(\beta_{1}-\alpha_{0}\right)^{2} & =\inf \frac{\int_{0}^{1}\left[\alpha_{0}+\left(\beta_{1}-\alpha_{0}\right) \sigma\right]^{n} V^{\prime}(\sigma)^{2} d \sigma}{\int_{0}^{1}\left[\alpha_{0}+\left(\beta_{1}-\alpha_{0}\right) \sigma\right]^{n} \mathcal{F}(H(\sigma)) V(\sigma)^{2} d \sigma} \\
& \leq \frac{2^{n-1}}{\mathcal{F}(B)} \inf \frac{\int_{0}^{1} \alpha_{0}^{n} V^{\prime}(\sigma)^{2} d \sigma+\int_{0}^{1} \sigma^{n}\left(\beta_{1}-\alpha_{0}\right)^{n} V^{\prime}(\sigma)^{2} d \sigma}{\int_{0}^{1} \alpha_{0}^{n} V(\sigma)^{2} d \sigma+\int_{0}^{1} \sigma^{n}\left(\beta_{1}-\alpha_{0}\right)^{n} V(\sigma)^{2} d \sigma} \\
& \leq \frac{2^{n-1}}{\mathcal{F}(B)} \max \left[\pi^{2}, \mu_{N}\right],
\end{aligned}
$$

where the infimum is taken over all $V \in C^{1}[0,1]$ such that $V(0)=V(1)=0$. Since $\beta_{0}-\alpha_{0}<\beta_{1}-\alpha_{0}$, we have proved (5).

Next we need to obtain an estimate on the slope of $u$ in terms of its slope at $\beta$.

Definition. Let $F_{+}$denote the restriction of $F$ to $(0, \infty)$, and let $G_{B k}, k>0$, denote the restriction of $F(u)-k f(B) u$ to $\left[f^{-1}(k f(B)), \infty\right)$ (See the definition above Lemma 1). Both $F_{+}^{-1}$ and $G_{B k}^{-1}$ are well defined on $(0, \infty)$ (see Lemma 2(v)).

Lemma 5. Let $u$ be as in Lemma 4 with $u(\alpha)=u(\beta)=0$ and $u^{\prime}(\beta)=-J$. Assume $J^{2}<2 F\left(J^{2} / 2 B\right)$. For sufficiently large $B$ we have:

(i) $u$ has a unique local extremum on $(\alpha, \beta)$.

(ii) $\sqrt{J^{2}-2 f(B) F_{+}^{-1}\left(\frac{1}{2} J^{2}\right)} \leq u^{\prime}(\alpha) \leq(\beta / \alpha)^{n} J+(\beta / \alpha)^{n} \sqrt{2 f(B) G_{B k}^{-1}\left(\frac{1}{2} k J^{2}\right)}$, where $k=(\beta / \gamma)^{2 n}$.

(iii) $\beta-\alpha \leq B / J+B / \sqrt{J^{2}-2 f(B) F_{+}^{-1}\left(J^{2} / 2\right)}+\left[2^{(n-1) / 2} / \sqrt{f^{\prime}(B)}\right] \max \left(\pi, \sqrt{\mu_{N}}\right)$.

Proof. We first observe that $u^{\prime \prime}>0$ whenever $u \leq u_{B}$ and $u^{\prime}<0$. This means that there are points $\gamma, \tilde{\beta} \in(\alpha, \beta), \alpha<\gamma<\tilde{\beta}<\beta$, such that $u(\gamma)>u_{B}(\gamma), u^{\prime}(\gamma)=0$, $u^{\prime}<0$ on $(\gamma, \beta)$, and $\tilde{\beta}$ is the only point in $(\gamma, \beta)$ where $u(\tilde{\beta})=u_{B}(\gamma)$. Moreover, we see that $u^{\prime \prime}(\gamma)=f(B) \varphi(\gamma)-\psi(r, u(\gamma))-f(u(\gamma))<0$ since $u(\gamma)>u_{B}(\gamma)$, and that $\left|u^{\prime}(r)\right| \geq J$ on $(\tilde{\beta}, \beta)$. Since $u_{B}(\tilde{\beta}) \leq B$ we have

$$
\beta-\tilde{\beta} \leq \frac{B}{J} .
$$

Because $u^{\prime \prime}(r)<0$ whenever $u^{\prime}(r)=0$ and $u(r)>u_{B}(r)$, it is not possible for $u$ to have any local minimum above $u_{B}$. This means that there is a point $\tilde{\alpha}<\gamma$ such that $u(\tilde{\alpha})=u_{B}(\tilde{\alpha})$ and $u(r)$ is nondecreasing on $(\tilde{\alpha}, \gamma)$. We want to show that $u$ is in fact increasing on $(\alpha, \gamma)$. This can be done by excluding the possibility that $u^{\prime}\left(r_{0}\right)=0$ for some $r_{0} \in(\alpha, \gamma)$. Suppose $r_{0}$ is the largest such value. Certainly $u\left(r_{0}\right) \leq u_{B}\left(r_{0}\right) \leq 0$, and $u^{\prime}(r) \geq B$ on $\left(r_{0}, \gamma\right)$. Hence $E\left(r_{0}\right) \geq E(\gamma)$. Using Lemma 2(ii)-(iii), we get

$$
\begin{aligned}
\frac{1}{2} u^{\prime}\left(r_{0}\right)^{2} & \geq F(u(\gamma))-f(B) u(\gamma)+f(B) u\left(r_{0}\right)-F\left(u\left(r_{0}\right)\right) \\
& \geq F(u(\gamma))-f(B) u(\gamma) .
\end{aligned}
$$

But since $\mathcal{E}(\gamma) \geq \mathcal{E}(\beta)$ we also have

$$
F(u(\gamma)) \geq \frac{1}{2} J^{2} \quad \text { or } \quad u(\gamma) \geq F_{+}^{-1}\left(\frac{1}{2} J^{2}\right),
$$


so that by Lemma $2(\mathrm{v})$

$$
\frac{1}{2} u^{\prime}\left(r_{0}\right)^{2} \geq \frac{1}{2} J^{2}-f(B) F_{+}^{-1}\left(\frac{1}{2} J^{2}\right) .
$$

Hence we only need to ensure that the right-hand side is positive. But this follows from $F\left(J^{2} / 2 f(B)\right)>\frac{1}{2} J^{2}$. This proves (i) as well as the first inequality in (ii). Also, since

$$
u^{\prime}(r) \geq \sqrt{J^{2}-2 f(B) F_{+}^{-1}\left(\frac{1}{2} J^{2}\right)} \quad \text { on }(\alpha, \gamma)
$$

we see that

$$
\tilde{\alpha}-\alpha \leq B / \sqrt{J^{2}-2 f(B) F_{+}^{-1}\left(\frac{1}{2} J^{2}\right)} .
$$

Combining this with (7) and (6), we obtain (iii).

It remains to prove the second inequality in (ii). We have

$$
\left(r^{n} u^{\prime}\right)\left(r^{n} u^{\prime}\right)^{\prime}=(f(B) \phi-\psi) r^{2 n} u^{\prime}-r^{2 n} F(u)^{\prime} .
$$

Integrating from $\alpha$ to $\gamma$, we have

$$
\begin{aligned}
-\frac{1}{2} \alpha^{2 n} u^{\prime}(\alpha)^{2} & =\int_{\alpha}^{\gamma}(f(B) \phi-\psi) r^{2 n} u^{\prime}-\left.r^{2 n} F(u)\right|_{\alpha} ^{\gamma}+\int_{\alpha}^{\gamma} 2 n r^{2 n-1} F(u) d r \\
& \geq-\gamma^{2 n} F(u(\gamma)) .
\end{aligned}
$$

Integrating (10) from $\gamma$ to $\beta$, we have

$$
\begin{aligned}
\frac{1}{2} \beta^{2 n} J^{2} & =\int_{\gamma}^{\beta}(f(B) \phi-\psi) r^{2 n} u^{\prime}-\int_{\gamma}^{\beta} r^{2 n} F(u)^{\prime} d r \\
& \geq-f(B) \beta^{2 n} u(\gamma)+\gamma^{2 n} F(u(\gamma))
\end{aligned}
$$

or

$$
\frac{1}{2}\left(\frac{\beta}{\gamma}\right)^{2 n} J^{2} \geq G_{B k}(u(\gamma))=F(u(\gamma))-k f(B) u(\gamma)
$$

where $k=(\beta / \gamma)^{2 n} \cdot G_{B k}^{-1}$ is well defined and increasing on $[0, \infty)$, so that we may write

$$
u(\gamma) \leq G_{B k}^{-1}\left(\frac{1}{2} k J^{2}\right)
$$

Combining (10) and (11), we have

$$
\frac{1}{2} \alpha^{2 n} u^{\prime}(\alpha)^{2} \leq \frac{1}{2} \beta^{2 n} J^{2}+f(B) \beta^{2 n} u(\gamma)
$$

so that

$$
u^{\prime}(\alpha)^{2} \leq\left(\frac{\beta}{\alpha}\right)^{2 n} J^{2}+2 f(B)\left(\frac{\beta}{\alpha}\right)^{2 n} G_{k}^{-1}\left(\frac{1}{2} k J^{2}\right) .
$$




\section{B. Estimates ON INTERVAls WHERE THE SOLUTION IS NEGATIVE}

In this section we will investigate solutions on $(0,1)$ that are negative on $(\alpha, \beta)$ with $u(\alpha)=u(\beta)=0$, and obtain results like those obtained in the previous section. Suppose that $u$ has a critical point at $\gamma \in(\alpha, \beta)$. Then

$$
u^{\prime \prime}(\gamma)=f(B) \varphi(\gamma)-\psi(\gamma, u(\gamma))-f(u(\gamma)) \geq f(B) d-\|\psi\|>0 .
$$

Hence there must be a single extremum at some point $\gamma$ in $(\alpha, \beta)$, namely a minimum. Since $u^{\prime} \leq 0$ on $(\alpha, \gamma)$ we see that $u^{\prime \prime}(r)>0$ there, and consequently

$$
|u(\gamma)| \leq\left|u^{\prime}(\alpha)\right|(\gamma-\alpha) \text {. }
$$

Lemma 6. Suppose $u$ is a solution of $(1)$ on $(0,1)$ and $u$ is negative on $(\alpha, \beta)$ with $u(\alpha)=u(\beta)=0$. Then

(i) $u$ has a single extremum (a minimum) in $(\alpha, \beta)$.

(ii) $\left\{2 F\left(\left(\sqrt{f(B)^{2}+\mu_{N} J^{2}}-f(B)\right) / \mu_{N}\right)\right\}^{1 / 2} \leq\left|u^{\prime}(\alpha)\right| \leq \frac{3}{2}(\beta / \alpha)^{n} J$.

(iii) $\beta-\alpha<\Psi_{n}\left(J /\left[\beta f\left(B_{*}\right)\right]\right)$, where $\Psi_{n} \in C^{1}[0,1)$ with $\Psi_{n}(0)=0$, $\Psi_{n}^{\prime}(0)=$ $2 \beta /(n+1), \Psi_{n}(s)>0$ on $(0,1)$ and $\Psi_{n}^{\prime}(s) \geq 0$ on $[0,1)$.

Proof. (i) follows from the above discussion. To prove (iii) we first look at the case $n>1$. Integrating the inequality

$$
\left(r^{n} u^{\prime}\right)^{\prime} \geq\left(f\left(B_{*}\right)-f(u)\right) r^{n} \geq f\left(B_{*}\right) r^{n}
$$

with respect to $r$, dividing by $r^{n}$, and integrating once more, we obtain

$$
u(r) \geq\left[\frac{f\left(B^{*}\right)}{n+1}-\frac{u^{\prime}(\beta)}{\beta}\right] \frac{\beta^{n+1}}{n-1}\left(r^{1-n}-\beta^{1-n}\right)-\frac{f\left(B_{*}\right)}{2(n+1)}\left(\beta^{2}-r^{2}\right) .
$$

Hence the right hand side of this inequality must be negative on $(\alpha, \beta)$. This implies, upon dividing by $f\left(B_{*}\right)[\beta-r] r /(n+1)$ and defining $\mu=\beta / r$ and $\kappa=$ $(n+1) u^{\prime}(\beta) /\left(f\left(\beta_{*}\right) \beta\right)$, that

$$
(1-\kappa) \frac{\mu^{n}+\mu^{n-1}+\cdots+\mu^{2}}{n-1}<\frac{\mu+1}{2} \text { if } 1<\mu<\beta / \alpha .
$$

Define

$$
\psi_{n}(\mu)=1-\frac{(n-1)(\mu+1)}{2\left(\mu^{2}+\mu^{3}+\cdots+\mu^{n}\right)},
$$

so that inequality (13) becomes

$$
\kappa>\psi_{n}(\mu), \quad \mu>1,
$$

or

$$
\min (\kappa, 1)>\psi_{n}(\mu)
$$

Note that $\psi_{n}(1)=0, \psi_{n}^{\prime}(\mu) \geq 0$ if $\mu>1, \psi_{n}$ maps $[1, \infty)$ one-to-one onto $[0,1)$, and if $r=\alpha$ then

$$
\frac{\beta-\alpha}{\beta}<\frac{\beta-\alpha}{\alpha}=\mu-1<\psi_{n}^{-1}\left[\min \left(1, \frac{(n+1) u^{\prime}(\beta)}{f\left(B_{*}\right) \beta}\right]-1\right.
$$

or, more simply,

$$
\beta-\alpha<\Psi_{n}\left(\frac{(n+1) u^{\prime}(\beta)}{f\left(B_{*}\right) \beta}\right):=\beta\left\{\psi_{n}^{-1}\left[\min \left(1, \frac{(n+1) u^{\prime}(\beta)}{f\left(B_{*}\right) \beta}\right)\right]-1\right\} .
$$


From our definition of $\Psi_{n}$ it follows that

$$
\Psi_{n}^{\prime}(0)=\beta / \psi_{n}^{\prime}\left(\psi_{n}^{-1}(0)\right)=\beta / \psi_{n}^{\prime}(1)=2 \beta /(n+1) .
$$

Next we consider the case $n=1$. Proceeding as before, we obtain

$$
u(r) \geq\left[u^{\prime}(\beta) \beta-\frac{1}{2} f\left(B_{*}\right) \beta^{2}\right] \ln \frac{r}{\beta}+\frac{1}{4} f\left(B_{*}\right)\left(r^{2}-\beta^{2}\right) .
$$

Defining $\kappa=2 u^{\prime}(\beta) \beta / f\left(B_{*}\right)$ and $\mu=\beta / r$, we have

$$
0>r^{-2} u(r) \geq \frac{1}{2} f\left(B_{*}\right)\left\{(1-\kappa) \mu^{2} \ln \mu+\frac{1}{2}\left(1-\mu^{2}\right)\right\}, \quad \mu \geq 1 .
$$

Let $S_{\kappa}(\mu)$ denote the expression in the braces. It is easy to see that $S_{\kappa}(1)=$ $0, S_{\kappa}^{\prime}(1)<0$ and $S_{\kappa}^{\prime \prime}=2(1-\kappa) \ln \mu+2-3 \kappa>0$ if $\kappa<2 / 3, \mu \geq 1$, while $\lim _{\mu \rightarrow \infty} S_{\kappa}(\mu)=\infty$. Therefore $S_{\kappa}(\mu)$ has a unique root $\mu_{1}(\kappa)$ on $(1, \infty)$ whenever $\kappa<2 / 3$. Actually the restriction $\kappa<2 / 3$ can be removed, since if $S_{\kappa}$ has more than two roots on $[1, \infty)$ then it must have at least 4 roots (counting multiplicity). But this implies the existence of a root for $S_{\kappa}^{\prime \prime \prime}(\mu)=2(1-\kappa) / \mu \neq 0$ for $\kappa \neq 1$. We again have

$$
\frac{\beta-\alpha}{\beta} \leq \frac{\beta-\alpha}{\alpha}=\mu-1<\mu_{1}(\kappa)-1,
$$

and as $\kappa \rightarrow 0$ the function $S_{\kappa}$ approaches the increasing function $S_{0} \equiv \mu^{2} \ln \mu+$ $\frac{1}{2}\left(1-\mu^{2}\right)$ on $[1, \infty)$, which has a double root at 1 . Hence $\beta-\alpha<\beta\left[\mu_{1}(\kappa)-1\right] \rightarrow 0$ as $\kappa \rightarrow 0$. We may define $\Psi_{1}(s)=\beta\left[\mu_{1}(2 s)-1\right]$. Writing $S_{\kappa}(\mu) \equiv S(\kappa, \mu)$ and differentiating $S(\kappa, \mu)$ implicitly, we get

$$
\frac{d \mu_{1}}{d \kappa}=-\frac{\partial S / \partial \kappa}{\partial S / \partial \mu}=\frac{\mu^{2} \ln \mu}{\partial S / \partial \mu}>0
$$

since $\partial S / \partial \mu$ is positive at the root $\mu_{1}$. Also we may obtain

$$
\frac{d \mu_{1}}{d \kappa}=\frac{\mu^{3} \ln \mu}{\mu^{2}-1} \longrightarrow \frac{1}{2} \quad \text { as } \quad \mu \longrightarrow 1
$$

Hence $\Psi_{1}^{\prime}(0)=\beta$. Next we use the energy estimates again: $E(\gamma) \geq E(\beta)=\frac{1}{2} J^{2}$ or, using $\mathrm{H}_{4}$,

$$
f(B)|u(\gamma)|+\frac{1}{2} \mu_{N}|u(\gamma)|^{2} \geq F(u(\gamma))-f(B) u(\gamma) \geq \frac{1}{2} J^{2}
$$

This implies that

$$
|u(\gamma)| \geq\left[\sqrt{f(B)^{2}+\mu_{N} J^{2}}-f(B)\right] / \mu_{N} .
$$

But $\mathcal{E}(\alpha) \geq \mathcal{E}(\gamma)=F(u(\gamma))$, so

$$
\left|u^{\prime}(\alpha)\right|>\left\{2 F\left(\left(\sqrt{f(B)^{2}+\mu_{N} J^{2}}-f(B)\right) / \mu_{N}\right)\right\}^{1 / 2} .
$$

If $J \ll f(B)$ we may write

$$
\left|u^{\prime}(\alpha)\right|>\left[2 F\left(\frac{J^{2}}{2 f(B)}\right)\right]^{1 / 2} \geq J \sqrt{\delta_{n} f\left(\frac{J^{2}}{2 f(B)}\right) / f(B)} .
$$

To obtain an upper bound for $\left|u^{\prime}(\alpha)\right|$ we integrate $(9)$ over $(\alpha, \beta)$ :

$$
\begin{aligned}
\frac{1}{2} \beta^{2 n} J^{2}-\frac{1}{2} \alpha^{2 n} u^{\prime}(\alpha)^{2} & \geq \int_{\alpha}^{\gamma} f(B) r^{2 n} u^{\prime} d r+\int_{\alpha}^{\beta} 2 n r^{2 n-1} F(u) d r \\
& \geq \gamma^{2 n} f(B) u(\gamma) .
\end{aligned}
$$


Hence

$$
\begin{aligned}
\left|u^{\prime}(\alpha)\right| & \leq\left(\frac{\beta}{\alpha}\right)^{n}\left[J^{2}+\frac{f(B)}{\mu_{N}}\left(\sqrt{f(B)^{2}+\mu_{N} J^{2}}-f(B)\right)\right]^{1 / 2} \\
& \leq\left(\frac{\beta}{\alpha}\right)^{n} J\left\{1+\frac{f(B)^{2}}{\mu_{N} J^{2}}\left(\sqrt{1+\frac{\mu_{N} J^{2}}{f(B)^{2}}}-1\right)\right\}^{1 / 2} \\
& \leq \frac{3}{2}\left(\frac{\beta}{\alpha}\right)^{n} J
\end{aligned}
$$

Lemma 7. Let $u$ be as in Lemma 6. Then

$$
\beta-\alpha>\left[\frac{f(B)}{J}+\mu_{N}\right]^{-1}, \quad \text { where } J=u^{\prime}(\beta) .
$$

Proof. We have $0 \leq r^{n}(f(B)-f(u))=\left(r^{n} u^{\prime}\right)^{\prime}$ if $\alpha \leq r \leq \beta \leq 1$, so $r^{n} u^{\prime}(r) \leq$ $\beta^{n} u^{\prime}(\beta)$ or $u^{\prime}(r) \leq J \beta^{n} r^{-n}$. If $n=1$, then integrating from $r$ to $\beta$ we get $-u(r) \leq$ $J \beta \ln (\beta / r)$. Since $f(u) \geq \mu_{N} u$ when $u \leq 0$, we have

$$
\left(r u^{\prime}\right)^{\prime} \leq r\left[f(B)+\mu_{N} J \beta \ln (\beta / r)\right] .
$$

Integrating once again from $r$ to $\beta$, we have

$$
\begin{aligned}
\beta u^{\prime}(\beta)-r u^{\prime}(r) \leq & {\left[f(B)+\mu_{N} J \beta \ln \beta\right]\left(\beta^{2}-r^{2}\right) / 2 } \\
& -\mu_{N} J \beta\left[\frac{1}{2} \beta^{2} \ln \beta-\frac{1}{2} r^{2} \ln r\right]+\frac{\mu_{N} J \beta}{4}\left(\beta^{2}-r^{2}\right) \\
\leq & \frac{1}{2}\left[f(B)+\mu_{N} J \beta \ln \beta+\frac{1}{2} \mu_{N} J \beta\right]\left(\beta^{2}-r^{2}\right) .
\end{aligned}
$$

Let $\alpha<\gamma<\beta$, where $u^{\prime}(\gamma)=0$; then

$$
\beta J \leq \frac{1}{2}\left[f(B)+\frac{1}{2} \mu_{N} J \beta\right] 2 \beta,(\beta-\gamma),
$$

so

$$
(\beta-\gamma) \geq J\left[f(B)+\frac{1}{2} \mu_{N} J \beta\right]^{-1}>J\left[f(B)+\mu_{N} J\right]^{-1} .
$$

If $n>1$, then, integrating $u^{\prime}(r) \leq J \beta^{n} r^{-n}$ from $r$ to $\beta$,

$$
-u(r) \leq J \beta^{n}\left[\beta^{1-n}-r^{1-n}\right] /(1-n),
$$

and since $f(u) \geq \mu_{N} u$ when $u \leq 0$ we have

$$
\left(r^{n} u^{\prime}\right)^{\prime} \leq r^{n}\left[f(B)+\mu_{N} J \beta^{n}\left(r^{1-n}-\beta^{1-n}\right) /(n-1)\right] .
$$

Integrating once again, we get

$$
\begin{aligned}
\beta^{n} u^{\prime}(\beta)-r^{n} u^{\prime}(r) \leq & {\left[f(B)-\mu_{N} J \beta^{n} /(n-1)\right]\left(\beta^{n+1}-r^{n+1}\right) /(n+1) } \\
& +\mu_{N} J \beta^{n}\left(\beta^{2}-r^{2}\right) / 2(n-1) .
\end{aligned}
$$

Setting $r=\gamma$, we have

$$
\begin{aligned}
\beta^{n} J \leq & {\left[f(B)-\mu_{N} J \beta^{n} /(n-1)\right]\left(\beta^{n+1}-\gamma^{n+1}\right) /(n+1) } \\
& +\mu_{N} J \beta^{n}\left(\beta^{2}-\gamma^{2}\right) / 2(n-1) \\
\leq & f(B) \beta^{n}(\beta-\gamma)+\mu_{N} J \beta^{n+1}(\beta-\gamma) /(n-1),
\end{aligned}
$$


so that

$$
\begin{aligned}
J & \leq \beta^{n}\left[f(B)+\mu_{N} J \beta /(n-1)\right](\beta-\gamma) \\
& \leq\left[f(B)+\mu_{N} J /(n-1)\right](\beta-\gamma)
\end{aligned}
$$

and

$$
\begin{aligned}
(\beta-\gamma) & \geq\left[f(B) / J+\mu_{N} /(n-1)\right]^{-1} \\
& \geq\left[f(B) / J+\mu_{N}\right]^{-1}
\end{aligned}
$$

Remark 3. It is easy to see that the function on the right side of this inequality is decreasing with respect to $J$, so that we have $\beta-\alpha \geq 1 / \sqrt{\mu_{N}}$ for all $J$. However, the following lemma shows that when $J$ becomes large we must in fact have $\beta-\alpha>1$. In particular, the solution to (1) with $\left|u^{\prime}(1)\right|$ large enough will not have any "loops" below the axis. We will make use of this fact in the proof of the main result.

Let $\theta(r, u):=\psi(r, u)+f(u)$, and let $D$ be a positive number such that $\theta_{u}(r, u)<$ $\mu_{N}$ whenever $u \leq-D$.

Lemma 8. Let $u$ be a solution of (1) on $R^{1}$.

(i) If there exists $0<\alpha<\beta$ such that $\alpha<1, u(\alpha)=u(\beta)=-D$, and $u<-D$ on $(\alpha, \beta)$, then if $n>1$ (resp. $n=1$ ) there exists a number $m_{*}>0$, independent of $\alpha$, such that $\beta>1$ whenever $u^{\prime}(\alpha)<-m_{*} / \alpha$ (resp. $u^{\prime}(\alpha)<m_{*} \ln (\alpha)$ ).

(ii) Let $u$ and $v$ be solutions of (1) such that $u<-D$ and $v<-D$ on $(\alpha, \beta)$, and $u(\alpha)=u(\beta)=v(\alpha)=v(\beta)=-D$. Then $u \equiv v$.

(iii) There exists a number $m_{* *}$ such that if $u$ is a solution on $[0,1]$ with $u^{\prime}(1)<$ $m_{* *}$, then $u<0$ on $(0,1)$ and $u(r) \rightarrow-\infty$ as $r \downarrow 0$.

Proof. Let $v=u+D$; then

$$
\begin{gathered}
\left(r^{n} v^{\prime}\right)^{\prime}=r^{n}(f(B) \phi-\psi(r, v-D)-f(v-D), \\
v(\alpha)=v(\beta)=0 .
\end{gathered}
$$

Suppose conclusion (i) of the lemma is false. Then there exist solutions $\left\{v_{i}\right\}_{i=1}^{\infty}$ to this problem with $v_{i}\left(\alpha_{i}\right)=v_{i}\left(\beta_{i}\right)=0$, where $0<\alpha_{i}<\beta_{i} \leq 1$ for all $i$, and $v_{i}^{\prime}\left(\alpha_{i}\right) \alpha_{i} \rightarrow-\infty\left(v_{i}^{\prime}\left(\alpha_{i}\right) \ln \left(\alpha_{i}\right) \rightarrow \infty\right.$ if $\left.n=1\right)$. Let $\gamma_{i}$ denote the point in $\left(\alpha_{i}, \beta_{i}\right)$ where $v_{i}$ achieves a minimum. From now on we will assume that any function defined on $\left[\alpha_{i}, \beta_{i}\right]$ is extended by defining it to be zero elsewhere, and that the extension is denoted by the same symbol. It is not difficult to see that the functions $\nu_{i}$ must be uniformly bounded, for if this were not so, then, letting $w_{i}=v_{i} /\left\|v_{i}\right\|_{\infty}$, we would have

$$
\left(r^{n} w_{i}^{\prime}\right)=r^{n}\left\|v_{i}\right\|^{-1}\left[f(B) \phi-\theta\left(r, v_{i}-D\right)\right], \quad \alpha_{i}<r<\beta_{i} .
$$

However, since $\theta_{u}(r, u)<\mu_{N}$ whenever $u \leq-D$, we can find a $\delta>0$, independent of $v_{i}$, such that

$$
\|\psi\|_{\infty} \geq \theta\left(r, v_{i}-D\right) \geq \theta(r,-D)+\left(\mu_{N}-\delta\right) v_{i} .
$$

This means that

$$
\left(r^{n} w_{i}^{\prime}\right)^{\prime}=r^{n}\left\{\left\|v_{i}\right\|_{\infty}^{-1}\left[f(B) \phi-\theta(r,-\theta)-\eta_{i}(r) w_{i}(r)\right\},\right.
$$


where $\eta_{i}(r) \leq \mu_{N}-\delta$. Multiplying the above equation by $-w_{i}(r)$ and integrating from $\gamma_{i}$ to $\beta_{i}$, we have

$$
\begin{aligned}
& \mu_{N} \int_{\gamma_{i}}^{\beta_{i}} r^{n} w_{i}^{2} d r \leq \int_{\gamma_{i}}^{\beta_{i}} r^{n}\left(w_{i}^{\prime}\right)^{2} d r \\
& \quad \leq-\left\|v_{i}\right\|_{\infty}^{-1} \int_{\gamma_{i}}^{\beta_{i}} r^{n}[f(B) \varphi-\theta(r,-D)] w_{i} d r+\int_{\gamma_{i}}^{\beta_{i}} r^{n}\left(\mu_{N}-\delta\right) w_{i}^{2} d r
\end{aligned}
$$

For $i$ sufficiently large this will be a contradiction. Therefore we may assume $\left\|v_{i}\right\|_{\infty} \leq C$ for some constant $C$. This implies that for each $\epsilon>0$ we have $\sup \left\{\left|v_{i}^{\prime \prime}(r)\right|: \epsilon \leq r \leq \beta_{i}, i \geq 1\right\}=c_{\epsilon}<\infty$. The constant $c_{\epsilon}$ depends only on $\epsilon$, so the set $\left\{v_{i}\right\}_{i=1}^{\infty}$ is precompact in $C^{1}[\epsilon, 1]$ for each $0<\epsilon<1$. Furthermore, we may assume without loss of generality that $v_{i} \rightarrow v$ in $C^{2}[\epsilon, 1]$ for each $0<\epsilon<1$. Since $v$ is a solution of $(16)$ on $(0,1)$ and $f(B) \varphi-\theta(r,-D) \geq f\left(B_{*}\right)>0$, it follows that $v \not \equiv 0$ and $v_{i}^{\prime}(1) \rightarrow v^{\prime}(1)>0$. Next, let $z_{i}=v_{i} / v_{i}^{\prime}(1)$; then $z_{i} \rightarrow v / v^{\prime}(1)$ in $C[\epsilon, 1]$ for any $0<\epsilon<1$, and

$$
z_{i}^{\prime \prime}+\frac{n}{r} z_{i}^{\prime}=f(B) \varphi-\theta\left(r, v_{i}-D\right)=: F_{i}(r) .
$$

The functions $F_{i}(r)$ are of course uniformly bounded and continuous on $(0,1]$. Let

$$
C_{1}:=\sup _{i \geq 1}\left\|F_{i}\right\|_{\infty}
$$

and let $z_{i}=\hat{z}_{i}+\tilde{z}_{i}$, where $\hat{z}_{i}$ is the particular solution that satisfies $\hat{z}_{i}\left(\alpha_{i}\right)=$ $\hat{z}_{i}^{\prime}\left(\alpha_{i}\right)=0$. Since

$$
\hat{z}_{i}(r)=\int_{\alpha_{i}}^{r} \int_{\alpha_{i}}^{\tau}(\sigma / \tau)^{n} F_{i}(\sigma) d \sigma d \tau
$$

we see that $\left\|z_{i}^{\prime}\right\|_{\infty} \leq C_{1}$ and $\left\|z_{i}\right\|_{\infty} \leq \frac{1}{2} C_{1}$. The function $\tilde{z}_{i}$ satisfies the homogeneous equation and $z_{i}\left(\alpha_{i}\right)=0$. We may solve explicitly. Let

$$
\begin{gathered}
j(r):=r^{(1-n) / 2} J_{(n-1) / 2}(r), \\
y(r):=r^{(1-n) / 2} Y_{(n-1) / 2}(r) \quad \text { if } n \text { is odd, } \\
y(r):=r^{(1-n) / 2} J_{(1-n) / 2}(r) \quad \text { if } n \text { is even, }
\end{gathered}
$$

and let $W$ denote their Wronskian:

$$
W(r)=y(r) j^{\prime}(r)-y^{\prime}(r) j(r) .
$$

We have

$$
\tilde{z}_{i}(r)=\tilde{z}_{i}^{\prime}(1)\left[y\left(\alpha_{i}\right) j(r)-j\left(\alpha_{i}\right) y(r)\right] /\left[y\left(\alpha_{i}\right) j^{\prime}(1)-j\left(\alpha_{i}\right) y^{\prime}(1)\right] .
$$

Since $\left|z_{i}^{\prime}(1)\right|=\left|z_{i}^{\prime}(1)-\hat{z}^{\prime}(1)\right| \leq 1+C$, we may assume without loss of generality that $\left\{z_{i}^{\prime}(1)\right\}_{i=1}^{\infty}$ converges to some number $\tilde{\mu}$. However, $\alpha_{i} \tilde{z}^{\prime}\left(\alpha_{i}\right) \rightarrow-\infty$. On the other hand,

$$
\alpha_{i} \tilde{z}^{\prime}\left(\alpha_{i}\right)=\alpha_{i} \tilde{z}^{\prime}(1) W\left(\alpha_{i}\right) /\left[y\left(\alpha_{i}\right) j^{\prime}(1)-j\left(\alpha_{i}\right) y^{\prime}(1)\right] .
$$

Using Abel's formula we have $W(r)=r^{-n} W(1)$, so that

$$
\alpha_{i} z^{\prime}\left(\alpha_{i}\right)=W(1) \tilde{z}^{\prime}(1) \alpha_{i}^{1-n} /\left[y\left(\alpha_{i}\right) j^{\prime}(1)-j\left(\alpha_{i}\right) y^{\prime}(1)\right] .
$$


Using the well-known asymptotic formulas for the Bessel functions $J_{k}(r) \approx$ $2^{-k} r^{k} / \Gamma(k+1)$ and $Y_{k}(r) \sim-\left(\Gamma(k) 2^{k} / \pi\right) r^{-k}$ if $k \neq 0, Y_{0}(r) \sim(2 / \pi) \ln (r)$, we see that

$$
\begin{gathered}
\left|\alpha_{i} z^{\prime}\left(\alpha_{i}\right)\right|=O(1) \quad \text { if } n>1 \\
\left|\alpha_{i} \ln \left(\alpha_{i}\right) z^{\prime}\left(\alpha_{i}\right)\right|=O(1) \quad \text { if } n=1 .
\end{gathered}
$$

This contradicts the hypothesis in (i).

To prove (ii) we let $u-v=w$. Then

$$
\begin{aligned}
-\left(r^{n} w^{\prime}\right)^{\prime} & =r^{n}[\theta(r, u(r)-D)-\theta(r, v(r)-D)] \\
& =r^{n} \eta(r) w(r),
\end{aligned}
$$

where $\eta(r)<\mu_{N}-\delta$. Multiplying by $w$ and integrating from $\alpha$ to $\beta$, we have

$$
\mu_{N} \int_{\alpha}^{\beta} r^{n} w^{2} d r \leq \int_{\alpha}^{\beta} r^{n}\left(w^{\prime}\right)^{2} d r \leq\left(\mu_{N}-\delta\right) \int_{\alpha}^{\beta} r^{n} w^{2} d r
$$

so that $w \equiv 0$. The proof of the third part of the lemma uses the same ideas. If $u^{\prime}(1)$ is sufficiently large, then, in view of the fact that $E$ is decreasing on intervals where $u^{\prime} \geq 0$, it follows that $u$ must attain a local minimum below $-D$ before rising to the value 0 at $r=1$. Suppose $u$ does not stay below $-D$; then there exists $0<\alpha<\beta<1$ such that $u(\alpha)=u(\beta)=-D$. Carrying out a change of variables $v=u+D$ as before and using the variational characterization of the principal eigenvalue, we obtain a contradiction. In this argument $D$ can be replaced by any larger value, so we see that $u(r)$ must be an increasing function on $(0, \beta)$. If $u$ remains bounded from below, one readily verifies from the fact that $\left(r^{n} u^{\prime}\right)^{\prime}$ is bounded that either $u(r) \rightarrow-\infty$ as $r \downarrow 0$ or $u^{\prime}(r) \rightarrow 0$ as $r \downarrow 0$. The second option leads to a contradiction by applying the Sturm comparison theorem (as in the proof of Lemma 4) to $v:=u+D$ and the solution to

$$
\begin{gathered}
-\left(r^{n} \varphi^{\prime}\right)^{\prime}=r^{n}\left[\frac{\theta(r, v-D)-\theta(r,-D)}{v(r)} \varphi(r)\right], \\
\varphi(0)=v(0), \quad \varphi^{\prime}(0)=0,
\end{gathered}
$$

where the term in the brackets is replaced by a function $\eta(r) \leq \mu_{N}-\delta$. Note that

$$
-\left(r^{n} v^{\prime}\right)^{\prime}=r^{n} \eta(r) v+r^{n}[\theta(r,-D)-f(B) \varphi],
$$

where the term in the brackets is negative. Hence $\varphi$ will be zero at some point $\mu$ between zero and the first positive root of $v$. But this, once again, violates the variational characterization of the principal eigenvalue, since $\eta(r) \leq \mu_{N}-\delta$.

\section{The initial value problem with $u^{\prime}(1)=c \sqrt{B f(B)}$}

In this section we combine the results from the previous two sections to show that, given any positive integer $K$, there are positive numbers $B_{K}$ and $c_{K}$ such that any solution of (1) with $u(1)=0, u^{\prime}(1)= \pm c_{K} \sqrt{B f(B)}$ has at least $K$ roots on $(0,1)$ provided $B \geq B_{K}$. The choice of a large value of $B$ ensures lots of oscillations. This is precisely what one expects since $f^{\prime}(B)$ will be large. If we fix $B$ and increase $\left|u^{\prime}(1)\right|$, the number of roots decreases. This is, of course, also as expected in view of (15) and Lemma 8 (iii). Hence it will prove important for the order of magnitude of $\left|u^{\prime}(1)\right|$ not to exceed $\sqrt{B f(B)}$ by too much. For technical reasons we also need to avoid small slopes at $r=1$. Numerical experiments have shown that for simple cases such as $\psi \equiv 0, \varphi \equiv 1$, small slopes at $r=1$ do not have much effect on the 
number of roots in $(0,1)$. However, if $\varphi$ is not constant this is not true anymore, as can be seen from the following simple example. Let $\varphi(r)=1$ for $r \leq \epsilon$ and $\varphi(r)=1 / f(B)$ for $r \geq 2 \epsilon$. Let $\psi(r, 0)=1$ for $\epsilon \leq r \leq 1$. Then if $u^{\prime}(1)=0$ we must have $u \equiv 0$ on $[2 \epsilon, 1]$. However, for large values of $\left|u^{\prime}(1)\right|$ and $B$ there may be many roots on $[2 \epsilon, 1]$.

Lemma 9. Let $u$ be as in Lemma 5 with $J=c \sqrt{B f(B)}, c \geq c_{0}>\sqrt{2 \lambda_{n}}, \alpha \geq$ $\alpha_{0}>0$, where $\lambda_{n}$ is defined in Lemma 2 (vi). Then the hypotheses of Lemma 5 will be satisfied for sufficiently large $B$, and

(i)

$$
\begin{gathered}
\beta-\alpha \leq c^{-1} \sqrt{B / f(B)}+\left(c^{2}-\sqrt{2 \lambda_{n}} c\right)^{-1 / 2} \sqrt{B / f(B)} \\
+2^{(n-1) / 2} \max \left(\pi, \sqrt{\mu_{N}}\right) / f^{\prime}(B) .
\end{gathered}
$$

(ii) There exists $B_{0}>0$ such that

$$
\sqrt{1-\frac{\sqrt{2 \lambda_{n}}}{c}} J \leq\left|u^{\prime}(\alpha)\right| \leq 2 \alpha^{-n} J \quad \text { for all } B>B_{0} .
$$

Here $B_{0}$ depends only on $n, f, \phi, \psi, c_{0}$ and $\alpha$.

Proof. It follows immediately from the superlinearity of $f$ (and in particular from Lemma 2(iii)) that the hypotheses of Lemma 5 will be satisfied provided $B$ is sufficiently large. In that case $J^{2}-2 F(B) \geq\left(c^{2}-2\right) B f(B) \rightarrow \infty$ as $B \rightarrow \infty$. If we let $\epsilon=\sqrt{2 \lambda_{n}} / c$, then

$$
J^{2}-2 f(B) F_{+}^{-1}\left(J^{2} / 2\right)=(1-\epsilon) J^{2}+\left[\epsilon c^{2} B f(B)-2 f(B) F_{+}^{-1}\left(J^{2} / 2\right)\right] .
$$

The term in the brackets is nonnegative for large $B$ :

$$
\begin{aligned}
F\left(\epsilon c^{2} B / 2\right)-\frac{J^{2}}{2} & \geq \frac{1}{2} \delta_{n} \epsilon c^{2} B f\left(\epsilon c^{2} B / 2\right)-\frac{c^{2}}{2} B f(B) \\
& \geq \frac{1}{2} \epsilon c^{2} B \lambda_{n}^{-1}\left(\epsilon c^{2} / 2\right) f(B)-\left(c^{2} / 2\right) B f(B) \\
& \geq\left(c^{2} B / 2\right)\left[\epsilon^{2} \lambda_{n}^{-1} c^{2} / 2-1\right] f(B) \geq 0 .
\end{aligned}
$$

Therefore,

$$
J^{2}-2 f(B) F_{+}^{-1}\left(J^{2} / 2\right) \geq c\left(c-\sqrt{2 \lambda_{n}}\right) B f(B)
$$

and Lemma 5(iii) yield (i).

We may therefore conclude that $\beta-\alpha \rightarrow 0$ as $B \rightarrow \infty$. The above computation and Lemma 5(ii) also show that

By Lemma 2(vii)

$$
u^{\prime}(\alpha) \geq \sqrt{1-\sqrt{2 \lambda_{n}} / c} J
$$

$$
f(B) G_{B k}^{-1}\left(\frac{1}{2} k J^{2}\right) \leq\left(1+1 / \lambda_{n}\right) J
$$

This implies that the upper bound for $u^{\prime}(\alpha)$ may be replaced by

$$
\left(\frac{\beta}{\alpha}\right)^{n} J\left\{1-\sqrt{2}\left(1+1 / \lambda_{n}\right)\right\}
$$

This quantity in turn may be bounded from above by $2 \alpha^{-n} J$ provided $B$ is chosen sufficiently large. 
Lemma 10. Let $u$ be as in Lemma 6 with $J=c \sqrt{B f(B)}$ and $c_{1} \geq c>\sqrt{3 \mu_{N}}$ and $\beta \geq \beta_{0}>0$. Then:

(i) $\lim _{B \rightarrow \infty}(\beta-\alpha)=0$.

(ii) There exists a constant $B_{1}>0$ such that

$$
\sqrt{\frac{2 \delta_{n}}{3 \mu_{N}}} J \leq\left|u^{\prime}(\alpha)\right| \leq 3 \cdot 2^{n-1} J
$$

whenever $B \geq B_{1}$, where $B_{1}$ depends only on $n, f, \phi, \psi, c$ and $\beta_{0}$.

Proof. (i) follows immediately from Lemmas 6(iii) and 1(ii). Using (i), we can pick $B_{1}$ so large that whenever $B \geq B_{1}$ then $\beta-\alpha<\beta_{0} / 2$, so that $\beta / \alpha<2$, and we therefore obtain the upper bound in (ii) by using Lemma 6 . Let us also assume $B_{1}$ is sufficiently large so that $c_{1} \mu_{N} B / f(B)<1$ whenever $B \geq B_{1}$. In that case

$$
\begin{aligned}
\sqrt{f(B)^{2}+\mu_{N} J^{2}}-f(B) & =f(B)\left\{\sqrt{1+c^{2} \mu_{N} B / f(B)}-1\right\} \\
& \geq f(B) \cdot \frac{1}{3} c^{2} B / f(B)=\frac{1}{3} c^{2} B
\end{aligned}
$$

so that

$$
u^{\prime}(\alpha)^{2} \geq 2 F\left(\frac{c^{2} B}{3 \mu_{N}}\right) \geq 2 \delta_{n} c^{2} B f\left(\frac{c^{2} B}{3 \mu_{N}}\right) / 3 \mu_{N} .
$$

Since $c^{2} / 3 \mu_{N} \geq 1$, this last term is larger than

$$
2 \delta_{n} c^{2} B f(B) / 3 \mu_{N}=2 \delta_{n} J^{2} / 3 \mu_{N},
$$

and we are done.

Theorem 1. Given $0<\beta_{0}<1$ and any positive integer $K$, there exist constants $c_{K}$ and $B_{K}=B_{K}\left(c_{K}\right)$ such that the solutions to (1) with $u(1)=0$ and $u^{\prime}(1)=$ $\pm c_{K} \sqrt{B f(B)}$ have at least $K$ roots in $\left(\beta_{0}, 1\right)$ provided $B \geq B_{K}$.

Proof. Let $\gamma_{N}=\sqrt{3 \mu_{N}}$ and note that $\gamma_{N}>3$. Let $L$ be an integer such that $K \leq 2 L$ and define $\bar{c}=3^{L}\left(4 / \beta_{0}\right)^{n L} c_{K}$. Here $c_{K}$ is picked sufficiently large so that

$$
\left(1-\sqrt{2 \lambda_{n}} / c_{K}\right)^{L / 2}\left(2 \delta_{n} / 3 \mu_{N}\right)^{L / 2}>\gamma_{N} / c_{K} .
$$

Next we pick $B_{K}$ such that

$$
\Psi_{n}\left(\frac{\bar{c} \sqrt{B f(B)}}{\beta_{0} f\left(B_{*}\right)}\right)<\frac{1-\beta_{0}}{2 L}
$$

whenever $B \geq B_{K}$. We also pick $B_{K}$ so large that

$$
2 \sqrt{B / 9 f(B)}+2^{(n-1) / 2} \max \left(\pi, \sqrt{\mu_{N}}\right) / f^{\prime}(B)<\frac{1-\beta_{0}}{2 L}
$$

whenever $B \geq B_{K}$. Let us denote the roots of $u$ by $1>r_{1}>r_{2}>\cdots$, and let us first consider the case $u^{\prime}(1)=c_{K} \sqrt{B f(B)}$. From (18) and Lemma 6(iii) we see that

$$
1-r_{1} \leq \Psi_{n}\left(\frac{c_{K} \sqrt{B f(B)}}{B_{0} f\left(B_{*}\right)}\right) \leq \Psi_{n}\left(\frac{\bar{c} \sqrt{B f(B)}}{\beta_{0} f\left(B_{*}\right)}\right)<\frac{1-\beta_{0}}{2 L} .
$$

Let $u^{\prime}\left(r_{1}\right) / \sqrt{B f(B)}:=c_{K-1}$. From Lemma 10 we see that

$$
\sqrt{2 \delta_{n} / 3 \mu_{N}} c_{K} \leq c_{K-1} \leq 3 \cdot 2^{n-1} c_{K}
$$


Since

$$
\begin{array}{r}
3<\gamma_{N}<\left(1-\sqrt{2 \lambda_{n}} / c_{K}\right)^{L}\left(2 \delta_{n} / 3 \mu_{N}\right)^{L} c_{K}<\left(1-\sqrt{2} / c_{K}\right)^{1 / 2}\left(2 \delta_{n} / 3 \mu_{N}\right)^{1 / 2} c_{K} \\
\leq c_{K-1} \leq 3 \cdot 2^{n-1} c_{K}<3^{L}\left(4 / \beta_{0}\right)^{n L} c_{K}<\bar{c}
\end{array}
$$

we may apply Lemma 9(i):

$$
\begin{aligned}
r_{1}-r_{2} \leq & c_{K}^{-1} \sqrt{B / f(B)}+\left(c_{K-1}^{2}-\sqrt{2 \lambda_{n}} c_{K-1}\right)^{-1} \sqrt{B f(B)} \\
& +2^{(n-1) / 2} \max \left(\pi, \sqrt{\mu_{N}}\right) / f^{\prime}(B) \\
\leq & 2 \sqrt{B / 9 f(B)}+2^{(n-1) / 2} \max \left(\pi, \sqrt{\mu_{N}}\right) / f^{\prime}(B)<\frac{1-\beta_{0}}{2 L} .
\end{aligned}
$$

Therefore we have exhibited the existence of one complete cycle on $\left[r_{2}, 1\right], 1-r_{2}<$ $\left(1-\beta_{0}\right) / L$. We also may apply Lemma 9 (ii) to obtain

$$
\begin{aligned}
3 & <\gamma_{N}<\left(2 \delta_{n} / 3 \mu_{N}\right)^{L / 2}\left(1-\sqrt{2 \lambda_{n}} / \bar{c}\right)^{L / 2} \leq\left(2 \delta_{n} / 3 \mu_{N}\right)^{1 / 2}\left(1-\sqrt{2 \lambda_{n}} / \bar{c}_{k}\right)^{1 / 2} c_{K} \\
& \leq c_{K-2} \leq 3\left(4 / \beta_{0}\right)^{n} c_{K} \leq 3^{L}\left(4 / \beta_{0}\right)^{n L} c_{K} \leq \bar{c} .
\end{aligned}
$$

Suppose we have found consecutive roots $r_{2 j}<r_{2 j-1}<\cdots<r_{1}<1$, and suppose $u^{\prime}\left(r_{2 j}\right) / \sqrt{B f(B)}:=c_{K-2 j}$ and

$$
\left(2 \delta_{n} / 3 \mu_{N}\right)^{j / 2}\left(1-\sqrt{2 \lambda_{n}} / \bar{c}\right)^{j / 2} c_{K} \leq c_{K-2 j} \leq 3^{j}\left(4 / \beta_{0}\right)^{n j} c_{K} .
$$

Then $\gamma_{N} \leq c_{K-2 j} \leq \bar{c}$, so we may apply the above arguments with $c_{K}$ replaced by $c_{K-2 j}$ to show that there exist roots $r_{2 j+2}<r_{2 j+1}<r_{2 j}$ and $1-r_{2 j} \leq$ $(1+j)\left(1-\beta_{0}\right) / L$. If we define $c_{K-2(j+1)}:=u^{\prime}\left(r_{2 j+2}\right) / \sqrt{B f(B)}$, then

$$
\begin{aligned}
\gamma_{N} & <\left(2 \delta_{n} / 3 \mu_{N}\right)^{(j+1) / 2}\left(1-\sqrt{2 \lambda_{n}} / \bar{c}\right)^{(j+1) / 2} c_{K} \\
& \leq c_{K-2(j+1)} \leq 3^{j+1}\left(4 / \beta_{0}\right)^{n(j+1)} c_{K} \leq \bar{c} .
\end{aligned}
$$

These steps can be repeated until we have shown the existence of roots $1>r_{1}>$ $r_{2}>\cdots>r_{2 L}$ with $1-r_{2 L}<L\left(1-\beta_{0}\right) / L=1-\beta_{0}$, so $r_{2 L}>\beta_{0}$. The proof for the case $u^{\prime}(1)=-c_{K} \sqrt{B f(B)}$ proceeds almost identically.

Remark 4. If we keep $B$ fixed and increase $c_{K}$, then the distance between consecutive roots will increase and $u$ will lose roots. However, for all roots that remain to the right of $\beta_{0}$ the estimates for the slopes are still valid:

$$
\begin{gathered}
\left(1-\sqrt{2} / c_{K}\right)^{j / 2}\left(2 \delta_{n} / 3 \mu_{N}\right)^{j / 2} \sqrt{B f(B)} \leq u^{\prime}\left(r_{2 j}\right), \\
u^{\prime}\left(r_{2 j-1}\right) \leq 3^{j}\left(4 / \beta_{0}\right)^{n j} c_{K} .
\end{gathered}
$$

\section{The Initial Value Problem with $u(0)>0, u^{\prime}(0)=0$}

It turns out that proving the existence of solutions with arbitrary numbers of roots on $(0,1)$ and with $u^{\prime}(0)=0, u(0)>0$ and $u(1)=0$ is particularly tricky, because one has simultaneously the problems of the singularity at the origin and the difficulty of controlling the size of the solution when the nonlinearity is superlinear. We handle this by shooting from the origin (as well as from $r=1$ ). Therefore we will need to get estimates for solutions of the initial value problem with $u(0)>0$, $u^{\prime}(0)=0$.

A function $h \in L^{p}[\alpha, \beta]$ will be said to be nondecreasing if after modification on a set of measure zero we have $h(x) \leq h(y)$ whenever $x \leq y$. We may similarly 
define nonincreasing. If $h$ is nondecreasing, then for any $0<\delta<\beta-\alpha$ and $\alpha \leq x \leq y \leq \beta-\delta$

$$
\frac{1}{\delta} \int_{x}^{x+\delta} h(\sigma) d \sigma \leq \frac{1}{\delta} \int_{y}^{y+\delta} h(\sigma) d \sigma .
$$

The converse is also true; if the above inequality holds for all $0<\delta<\beta-\alpha$ and all $\alpha \leq x \leq y \leq \beta-\delta$ then $h$ is nondecreasing. We therefore easily deduce the following result.

Lemma 11. Let $\left\{h_{i}\right\}_{i=1}^{\infty} \subset L^{p}[\alpha, \beta]$, with $h_{i}$ nondecreasing for each $i$. Suppose $h_{i} \rightarrow h$ weakly in $L^{p}[\alpha, \beta]$. Then $h$ is also nondecreasing. Moreover, $h_{i}(x) \rightarrow h(x)$ on the set of points where $h$ is continuous (modulo a set of measure zero).

Proof. First we modify the functions $h_{i}$ so that $h_{i}(x) \leq h_{i}(y)$ for all $x \leq y$ and all $i$. We have

$$
\frac{1}{\delta} \int_{x-\delta}^{x} h_{i}(\sigma) d \sigma \leq h_{i}(x) \leq \frac{1}{\delta} \int_{x}^{x+\delta} h_{i}(\sigma) d \sigma
$$

Hence

$$
\begin{aligned}
h(x-\delta) & \leq \frac{1}{\delta} \int_{x-\delta}^{x} h(\sigma) d \sigma \leq \liminf _{i \rightarrow \infty} h_{i}(x) \leq \limsup _{i \rightarrow \infty} h_{i}(x) \\
& \leq \frac{1}{\delta} \int_{x}^{x+\delta} h(\sigma) d \sigma \leq h(x+\delta) .
\end{aligned}
$$

Since $\delta$ is arbitrary, we have the desired convergence at each point of continuity.

Remark 5. Note that the conclusion of Lemma 11 is of course still valid if we replace $h_{i}$ by $h_{i}+e_{i}$, where $h_{i}$ is nondecreasing and $e_{i} \rightarrow 0$ uniformly.

Lemma 12. Let $g:(0, \infty) \rightarrow(0, \infty)$ be a nondecreasing function and $Q(s):=$ $\int_{0}^{s} q(\sigma) d \sigma$. Let $U \in W_{2, l o c}^{2}\left(R^{N}\right) \cap C\left(R^{N}\right), N \geq 2$, be a radially symmetric solution of

$$
-\Delta U=q(U)
$$

with $U(0)=1$, and suppose $2 N Q(\sigma) \geq(N-2) q(\sigma) \sigma$ for all $\sigma \in(0,1]$, with strict inequality on a set of positive measure. Then $U$ is zero on the sphere $\partial B_{R}(0)$ for some $0<R$.

Proof. Suppose $U>0$ in $R^{N}$. Let $u(|x|)=U(x)$, so that $u^{\prime \prime}(r)+n u^{\prime}(r) / r=$ $-q(u)<0$ and $u(0)=1, u^{\prime}(0)=0, u(r)>0$ for all $r>0$. This implies that $u^{\prime}(r) \leq 0$ and $\lim _{r \rightarrow \infty} u(r)=0$. Hence, for each $\delta>0$ there is an $r_{\delta}>0$ such that $u\left(r_{\delta}\right)=\delta$ and $r_{\delta} \rightarrow \infty$ as $\delta \rightarrow 0$. Let $u_{\delta}:=u-\delta$; then

$$
\begin{gathered}
u_{\delta}^{\prime \prime}-\frac{n u_{\delta}^{\prime}}{r}=-q\left(\delta+u_{\delta}\right) \quad \text { in } \quad\left(0, r_{\delta}\right), \\
u_{\delta}^{\prime}(0)=0, \quad u_{\delta}\left(r_{\delta}\right)=0 .
\end{gathered}
$$

We may apply Pohožaev's identity [6], since the restriction in [6] that $q$ be continuous is not essential - all that is needed is that $q(u)$ be integrable, which is certainly the case here because $q(u(r))$ is monotone. Pohožaev's identity yields

$$
\int_{0}^{r_{\delta}}\left\{2 N[Q(u+\delta)-Q(\delta)]-(N-2) q\left(u_{\delta}+\delta\right) u_{\delta}\right\}\left(r / r_{\delta}\right)^{n} d r=u^{\prime}\left(r_{\delta}\right)^{2}
$$


If we now let $\delta \rightarrow \infty$ the left side will go to a positive limit and the right to zero, so we get a contradiction.

Consider the equation

$$
\begin{array}{cc}
-\left(w_{M}^{\prime \prime}+\frac{n}{r} w_{M}^{\prime}\right)=g\left(r, w_{M}(r)\right), & 0<r<1, \\
w_{M}(0)=M_{*}=: M+D, & w_{M}^{\prime}(0)=0,
\end{array}
$$

where $g(r, w):=f(w+D)-f(B) \phi(r)+\psi(r, w+D)$ for $w \geq 0$ and $g(r, w):=$ $g(r, 0)$ for $w<0$. Let us change variables: $s=\sqrt{\gamma\left(M_{*}\right) / M_{*}} r$, where $\gamma\left(M_{*}\right)=$ $\inf _{0 \leq r \leq 1} g(r, M)$ and where we will consider only the set of values $M$ such that $M_{*}=$ $M+D$ exceeds some fixed positive value $M_{* *}>B$. Let $v_{M}(s):=M_{*}^{-1} w_{M}(r)$; then

$$
v_{M}^{\prime \prime}+\frac{n}{s} v_{M}^{\prime}=\gamma\left(M^{*}\right)^{-1} g\left(\sqrt{M_{*} / \gamma\left(M_{*}\right)} s, M_{*} v_{M}(s)\right) .
$$

The two equations for $u_{M}$ and $v_{M}$ may be rewritten as Volterra equations. In case $N>2$, i.e., $n>1$, we have

$$
\begin{gathered}
w_{M}(r)=M_{*}-\frac{1}{n-1} \int_{0}^{r} \sigma\left[1-(\sigma / r)^{n-1}\right] g\left(\sigma, w_{m}(\sigma)\right) d \sigma \\
v_{M}(s)=1-\frac{1}{n-1} \int_{0}^{s} \sigma\left[1-(\sigma / s)^{n-1}\right] \gamma\left(M_{*}\right)^{-1} g\left(\sqrt{M_{*} / \gamma\left(M_{*}\right)} \sigma, M_{*} v_{M}(\sigma)\right) d \sigma .
\end{gathered}
$$

In case $N=2$,

$$
\begin{gathered}
u_{M}(r)=M_{*}-\int_{0}^{r} \sigma \ln (r / \sigma) g\left(\sigma, u_{M}(\sigma)\right) d \sigma \\
v_{M}(s)=1-\int_{0}^{s} \sigma \ln (s / \sigma) \gamma\left(M_{*}\right)^{-1} g\left(\sqrt{M_{*} / \gamma\left(M_{*}\right)} \sigma, M_{*} v_{M}(\sigma)\right) d \sigma .
\end{gathered}
$$

Let us first consider the case $n>1$. Since

$$
v_{M}^{\prime}(s)=-\int_{0}^{s}(\sigma / s)^{n} \gamma\left(M_{*}\right)^{-1} g\left(\sqrt{M_{*} / \gamma\left(M_{*}\right)} \sigma, M_{*} v_{M}(\sigma)\right] d \sigma
$$

we have

$$
\left|v_{M}^{\prime}(s)\right|<\int_{0}^{s}(\sigma / s)^{n}\left[\frac{1+\|\psi\| / f(B)}{1-f(B) / f\left(M_{* *}\right)}\right] d \sigma .
$$

Let $C_{*}$ denote the quantity in the brackets; then $\left|v_{M}^{\prime}(s)\right| \leq C_{*} s /(n+1) \leq C_{*} /(n+1)$. Also we have

$$
\left|v_{M}^{\prime \prime}(s)\right| \leq \frac{g\left(\sqrt{M_{*} / \gamma\left(M_{*}\right)} s, M_{*} v_{M}(s)\right)}{\gamma\left(M_{*}\right)}+\frac{n C_{*}}{n+1} \leq \frac{(2 n+1) C_{*}}{n+1} .
$$

By the Arzelà-Ascoli theorem, $\left\{v_{M}: M>M_{* *}\right\}$ is a precompact set in $C^{1}[0,1]$. Therefore we may pick a sequence $\left\{v_{M_{i}}\right\}$ such that $M_{i} \rightarrow \infty$ and $v_{M_{i}} \rightarrow v$ in $C^{1}[0,1]$. We may furthermore require that this sequence converges pointwise to $\limsup _{M \rightarrow \infty} v_{M}(s)$ for all $s \in \mathcal{Q} \cap[0,1]$, and also (using Lemma 11) that the uniformly bounded sequence $g\left(\sqrt{M_{i *}\left(\gamma\left(M_{i *}\right)\right.} s, M_{i *} v_{M_{i}}(s)\right) / \gamma\left(M_{i *}\right)$ converges weakly and pointwise almost everywhere to a function $\Gamma \in L_{2}[0,1]$. Note that $v^{\prime}(s) \leq 0$ and that $\Gamma$ is nonincreasing. Let $\sigma$ be any point in $[0,1)$; then there exists a unique $s \in(0,1]$ such that $v(s)=\sigma$. Modify $\Gamma$ on a set of measure zero so that 
$\Gamma\left(s_{1}\right) \leq \Gamma\left(s_{2}\right)$ whenever $s_{1} \leq s_{2}$. Define $\tilde{g}(\sigma)=\Gamma(s)$ so that $\tilde{g}(v(s)=\Gamma(s), \tilde{g}$ is nondecreasing and $V(x):=v(|x|) \in W_{2, l o c}^{2}\left(R^{N}\right)$ is a weak solution to the problem

$$
\Delta V+\tilde{g}(V)=0 .
$$

We see that $\underline{g}(u) \leq \tilde{g}(u) \leq \bar{g}(u)$, so that

$$
\frac{2 n+2}{n-1} \tilde{G}(u):=\frac{2 n+2}{n-1} \int_{0}^{u} \tilde{g}(\sigma) d \sigma \geq \frac{2 n+2}{n-1} \underline{G}(u) \geq \bar{g}(u) u \geq \tilde{g}(u) u,
$$

with strict inequality on a set of positive measure. But by the previous lemma this implies the existence of a positive number $R_{0}$ such that $v\left(R_{0}\right)=0$. Let $s_{0} \in \mathcal{Q} \cap$ $\left(R_{0}, 1\right]$; then $w_{M}\left(s_{0}\right) \leq 0$ for all sufficiently large $M$, and hence $u\left(\sqrt{M_{*} / \gamma\left(M_{*}\right)} R_{0}\right)$ $\leq 0$. We have proven:

Lemma 13. Let $v_{M}$ be a radially symmetric solution of $-\Delta v_{M}=g\left(|x|, v_{M}\right)$ in $R^{N}$ with $v_{M}(0)=M, M>B$. Let $r_{M}$ be the smallest positive number such that $v_{M}\left(r_{M}\right)=-D$ (if $v_{M}>0$ in $R^{N}$ we set $r_{M}=\infty$ ). Then there exist positive constants $c_{1}, c_{2}$ and $M_{1}$ such that

$$
\begin{aligned}
r_{M} & \leq c_{1} \sqrt{M / f(M)} \quad \forall M \geq M_{1}, \\
u_{M}^{\prime}\left(r_{M}\right) & \leq-c_{2} \sqrt{M \gamma_{*}(M)} \quad \forall M \geq M_{1} .
\end{aligned}
$$

The proof of (24) is an immediate consequence of the definition of $v_{M}$. The proof for the case $N=2$ proceeds similarly.

Remark 6. Pohožaev's identity was also used to tell us that $c_{2}:=v_{M}^{\prime}\left(r_{M}\right)>0$.

\section{An Overdetermined Boundary Value Problem}

The hypotheses $H_{1}$ and $H_{4}$ imply that there exists a positive number $D$ such that $f^{\prime}(u)<\mu_{N}$ whenever $u \leq-D$. In this section we consider the problem

$$
\begin{gathered}
w_{B}^{\prime \prime}+\frac{n w_{B}^{\prime}}{r}=f(B) \phi-\psi\left(r, w_{B}\right)-f\left(w_{B}\right):=-g\left(w_{B}, r\right), \\
w_{B}(0)=M>0, \quad w_{B}^{\prime}(0)=0, \quad w_{B}(\gamma)=-D, \quad w_{B}(\gamma)=0,
\end{gathered}
$$

where $0<\gamma<1$. We expect solutions only if $M$ and $\gamma$ are chosen in a special way.

Definition. Let $\bar{\mu}$ denote the principal eigenvalue for the problem

$$
\begin{aligned}
-\left(s^{n} w^{\prime}\right)^{\prime} & =\lambda s^{n} w(s), \quad \frac{1}{2}<s<1, \\
\varphi\left(\frac{1}{2}\right) & =0, \quad \phi^{\prime}(1)=0 .
\end{aligned}
$$

Let $w_{B}$ be a solution of $(25)$ such that $w_{B}(0)=M$ and $w_{B}^{\prime}(0)=0$. Let $\gamma_{B}$ denote the smallest positive value of $r$ where $w_{B}+D$ attains a negative local minimum. Once $B$ is fixed, we know from Lemma 13 that $w_{B}+D$ will attain negative values provided $M$ is chosen sufficiently large. It will be convenient to make the following adjustment of the value of $D$ (if needed): let $w_{*}$ denote the solution of $(25)$ with initial conditions $w_{*}(0)=B, w_{*}^{\prime}(0)=0$. If need be, increase the value of $D$ so that $-D<\min _{0 \leq r \leq 1} w_{*}(r)$. This means there must be a smallest value of $M$, denoted $M_{B}$, such that $w_{B}$ attains a local minimum of $-D$ at some point $\gamma_{B}: w_{B}\left(\gamma_{B}\right)=-D, w_{B}^{\prime}\left(\gamma_{B}\right)=0$. It should be noted that $w_{B}$ cannot have any local maximum below $u_{B}$ (hence below $B_{*}$ ), so that $w_{B}$ crosses the $r$-axis only once on $\left(0, \gamma_{B}\right)$. This is important since we need to keep control of the number of roots. 
Definition. $\nu_{B}=\min _{\sigma \geq B_{*} / 2} f^{\prime}(\sigma)$. We assume $B$ is chosen large enough so that $\nu_{B}>\left\|\psi_{u}\right\|$. For sufficiently large $B$ we of course have $\nu_{B}=f^{\prime}\left(B_{*} / 2\right)$.

Lemma 14. Suppose $u_{B}$ is the solution of (25)-(26) corresponding to the smallest possible value of $M$, denoted $M_{B}$. We have

$$
\gamma_{B} \leq\left[\max \left\{\frac{\bar{\mu}\left(1+2 D / B_{*}\right)}{\nu_{B}-\left\|\psi_{u}\right\|}, \frac{4 \mu_{N}}{\nu_{B}-\left\|\psi_{u}\right\|}\right\}\right]^{1 / 2} .
$$

In particular,

$$
\lim _{B \rightarrow \infty} \gamma_{B}=0
$$

Proof. Let $\beta_{B}$ be the unique number such that $u_{B}\left(\beta_{B}\right)=B_{*}$, with $u_{B}$ decreasing on $\left[\beta_{B}, \gamma_{B}\right]$. Let $\mu_{B}$ be the function that is defined as

$$
\mu_{B}(r):= \begin{cases}{\left[g\left(w_{B}(r), r\right)-g\left(B_{*}, r\right) /\left[w_{B}(r)-B_{*}\right]\right.} & \text { if } w_{B}(r) \neq B_{*}, \\ g_{u}\left(B_{*}, r\right) & \text { if } w_{B}(r)=B_{*} .\end{cases}
$$

Let $s=r / \beta_{B}$ and $v_{B}(s):=u_{B}\left(\beta_{B} s\right)-B_{*}$; then

$$
\begin{aligned}
-\left(s^{n} v_{B}^{\prime}\right)^{\prime} & =\beta_{B}^{2} s^{n} \mu_{B}\left(\beta_{B} s\right) v_{B}, \\
v_{B}^{\prime}(0) & =0, \quad v_{B}(1)=0 .
\end{aligned}
$$

Using the variational characterization of the principal eigenvalue, we obtain

$$
\beta_{B}^{2}=\inf \int_{0}^{1} s^{n} v^{\prime}(s)^{2} d s / \int_{0}^{1} s^{n} \mu_{B}\left(\beta_{B} s\right) v(s)^{2} d s,
$$

where the infimum is taken over all $v \in C^{1}[0,1]$ that satisfy $v(1)=0$. Therefore,

$$
\beta_{B}^{2} \leq \mu_{N} / \min \mu_{B}\left(\beta_{B} s\right) .
$$

We may find arbitrarily large $B$ such that $f^{\prime}(\sigma) \geq f^{\prime}\left(B_{*}\right)$ whenever $\sigma \geq B_{*}$. Then

$$
\beta_{B} \leq \sqrt{\mu_{N} /\left(\min _{s \geq B_{*}} f^{\prime}(s)-\left\|\psi_{u}\right\|\right)}
$$

If $\gamma_{B} \leq 2 \beta_{B}$, then

$$
\gamma_{B} \leq \sqrt{4 \mu_{N} /\left(\min _{s \geq B_{*}} f^{\prime}(s)-\left\|\psi_{u}\right\|\right)} ;
$$

otherwise we make another change of variables: $s=r / \gamma_{B}, v_{B}(s)=u_{B}\left(\gamma_{B} s\right)-B_{*}$, $\sigma_{B}:=\beta_{B} / \gamma_{B}$ :

$$
\begin{gathered}
-\left(s^{n} v_{B}^{\prime}\right)^{\prime}=\gamma_{B}^{2} s^{n} \mu_{B}\left(\gamma_{B} s\right) v_{B} \quad \text { if } \quad \sigma_{B}<s<1, \\
v_{B}\left(\sigma_{B}\right)=0, \quad v_{B}^{\prime}(1)=0 .
\end{gathered}
$$

Let

$$
C_{B}=\min _{\substack{D \leq \sigma \leq B_{*} \\ 0 \leq r \leq 1}} \mu_{B}(\sigma) .
$$


Once again, using the variational characterization of the principal eigenvalue we have, since $\sigma_{B} \leq 1 / 2$,

$$
\begin{aligned}
\gamma_{B}^{2} & =\inf \int_{\sigma_{B}}^{1} s^{n} v^{\prime}(s)^{2} d s / \int_{\sigma_{B}}^{1} s^{n} \mu_{B}\left(\gamma_{B} s\right) v(s)^{2} d s \\
& \leq C_{B}^{-1} \inf \int_{1 / 2}^{1} s^{n} v^{\prime}(s)^{2} d s / \int_{1 / 2}^{1} s^{n} v(s)^{2} d s=\bar{\mu} / C_{B} .
\end{aligned}
$$

If $B_{*} / 2 \leq \sigma \leq B_{*}$, then

$$
\left[g(\sigma, r)-g\left(B_{*}, r\right)\right] /\left[\sigma-B_{*}\right] \geq \nu_{B}-\left\|\psi_{u}\right\|,
$$

while if $-D \leq \sigma \leq B_{*} / 2$, then

$$
\left[g(\sigma, r)-g\left(B_{*}, r\right)\right] /\left(\sigma-B_{*}\right) \geq \frac{1}{2}\left[\nu_{B}-\left\|\psi_{u}\right\|\right] B_{*} /\left(B_{*} / 2+D\right),
$$

and hence

$$
C_{B} \geq \frac{\nu_{B}-\left\|\psi_{u}\right\|}{1+2 D / B_{*}}
$$

and

$$
\gamma_{B} \leq \sqrt{\frac{\bar{\mu}\left(1+2 D / B_{*}\right)}{\nu_{B}-\left\|\psi_{u}\right\|}} .
$$

Remark \%. Note that this estimate is independent of $M$.

\section{Proof of the Main Result}

Let $K$ be an arbitrary positive integer. By Theorem 1 we may choose $\underline{c}$ and $B$ sufficiently large so that the solution $u$ with $u^{\prime}(1)= \pm \underline{c} \sqrt{B f(B)}$ has at least $K+1$ roots, $\left\{r_{j}\right\}_{j=1}^{K+1}, 1>r_{1}>r_{2}>\cdots>r_{K+1}>\frac{1}{2}$. Moreover, we will pick the sign so that $u$ is negative on the interval $\left(r_{K+1}, r_{K}\right)$. Let $u_{c}$ denote the solution to (1) with $u_{c}^{\prime}(1)=(c / \underline{c}) u^{\prime}(1), c \geq \underline{c}$. As we increase $c, u_{c}$ will have to lose roots by virtue of Lemma 8. The loss of roots as we increase $c$ can happen in three ways: 1) two or more roots merge at some point in $(0,1) ; 2)$ two or more roots merge at the origin; $3)$ a root tends to zero while the next larger root stays away from the origin. The first possibility is excluded by Remark 4 . Lemma 7 precludes the roots $r_{K+1}$ and $r_{K}$ from merging at the origin (although the consecutive roots between which $u$ is positive may merge at the origin). Hence there must exist a value $c_{*}>\underline{c}$ such that $u_{c}$ has $K+1$ roots in $(0,1)$ if $c_{*}>c>\underline{c}$ but $u_{c_{*}}$ has only $K$ roots in $(0,1)$. We will show that $u_{*}$ is precisely the solution we are looking for: it has $K$ roots in $(0,1), u_{*}(0)<0$ and $u_{*}^{\prime}(0)=0$. To prove this, we transform the equation to the form $\left(3_{n}\right): s=r^{1-n}, u_{c}(r)=U(s), U^{\prime}(1)=\mp c \sqrt{B f(B)} /(n-1)$. Let us denote $r_{j}^{1-n}=s_{j}, j=1,2, \ldots, K+1$. Integrating $\left(3_{n}\right)$, we have

$$
U^{\prime}(s)=U^{\prime}\left(s_{K}\right)+\frac{1}{(n-1)^{2}} \int_{s_{K}}^{s}\left[h_{B}(\sigma, U(\sigma))-\sigma^{-2 n /(n-1)} f(U(\sigma))\right] d \sigma,
$$

where

$$
h_{B}(\sigma, U):=\left[f(B) \phi\left(\sigma^{-1 /(n-1)}\right)-\psi\left(\sigma^{-1 /(n-1)}, U(\sigma)\right)\right] \sigma^{-2 n /(n-1)} .
$$


Of course $U$ depends continuously on $c$. We write $s_{K}=s_{K}(c)$. Since $B>s_{0}$ (see Lemma 2 (i)), one easily verifies that $U^{\prime \prime}>0$ on $\left(s_{K}, s_{K+1}\right)$. Thus there exists a unique minimum at $\sigma_{K} \in\left(s_{K}, s_{K+1}\right)$ and $U^{\prime}\left(\sigma_{K}\right)=0$, and $\sigma_{K}$ also depends continuously on $c, \sigma_{K}=\sigma_{K}(c)$. Let $c$ increase to $c_{*}$. Since $s_{K}(c)$ stays bounded, we may find a sequence $c_{i} \uparrow c_{*}$ such that $s_{K}\left(c_{i}\right) \rightarrow s_{K}^{*}$ and $\sigma_{K}\left(c_{i}\right) \rightarrow \sigma_{K}^{*}$, where $\sigma_{K}^{*}$ is either finite or $+\infty$. We will use $U_{i}$ to denote the solution to $\left(3_{n}\right)$ with initial condition $\mp c_{i} \sqrt{B f(B)} /(n-1)$. Then

$$
\begin{gathered}
\left.0=U_{i}^{\prime}\left(s_{K}\left(c_{i}\right)\right)+\frac{1}{(n-1)^{2}} \int_{s_{K}\left(c_{i}\right)}^{\sigma_{K}\left(c_{i}\right)} h_{B}\left(\sigma, U_{i}(\sigma)\right)\right) d \sigma \\
-\frac{1}{(n-1)^{2}} \int_{s_{K}\left(c_{i}\right)}^{\sigma_{K}\left(c_{i}\right)} \sigma^{-2 n /(n-1)} f\left(U_{i}(\sigma)\right) d \sigma .
\end{gathered}
$$

The first integrand is absolutely integrable on $[0, \infty)$ and is in fact dominated by an absolutely integrable function $c s t \times \sigma^{-2 n /(n-1)}$. Hence, as $c_{i} \uparrow c_{*}$ the integral will converge to

$$
\frac{1}{(n-1)^{2}} \int_{s_{K}^{*}}^{\sigma_{K}^{*}} h_{B}\left(\sigma, U_{*}(\sigma)\right) d \sigma
$$

where $U_{*}$ is the solution with $U_{*}^{\prime}(1)=\mp c_{*} \sqrt{B f(B)} /(n-1)$.

Of course, $U_{i}^{\prime}\left(s_{K}\right) \rightarrow U_{*}^{\prime}\left(s_{K}^{*}\right)$ since the $\left|U_{i}^{\prime}\left(s_{K}\left(c_{i}\right)\right)\right|$ 's are uniformly bounded on bounded intervals. This also implies that the set of functions $\left\{U_{i}\right\}$ is uniformly bounded from below by a linear function:

$$
0 \geq U_{i}(\sigma) \geq-C_{1}\left[\sigma-C_{2}\right]
$$

where $C_{1}=\sup _{i}\left|U_{i}^{\prime}\left(s_{K}\left(c_{i}\right)\right)\right|$ and $C_{2}=\inf _{i} s_{K}\left(c_{i}\right)$. This implies

$$
-f(U(\sigma)) \sigma^{-2 n /(n-1)} \leq \mu_{N} C_{1}\left[\sigma-C_{2}\right] \sigma^{-2 n /(n-1)},
$$

so that we may apply Lebesgue's dominated convergence theorem to deduce that the second integral converges and

$$
0=U_{*}^{\prime}\left(s_{K}^{*}\right)+\frac{1}{(n-1)^{2}} \int_{S_{K}^{*}}^{\sigma_{K}^{*}}\left[h_{B}\left(\sigma, U_{*}(\sigma)\right)-\sigma^{-2 n /(n-1)} f\left(U_{*}(\sigma)\right)\right] d \sigma .
$$

If we define $u_{*}(r)=U_{*}(s)$ then $u_{*}$ is a solution of (1). Let us show that $\sigma_{K}^{*}=\infty$. If not, then $U_{*}^{\prime}(\sigma)$ would be positive for $\sigma>\sigma_{K}^{*}$, so that $U_{*}$ would have another root beyond $s_{K}^{*}$, a contradiction. We still need to verify that $U_{*}$ is bounded. Since we may write

$$
U_{*}^{\prime}(s)=\frac{-1}{(n-1)^{2}} \int_{s}^{\infty}\left[h_{B}\left(\sigma, U_{*}(\sigma)\right)-\sigma^{-2 n /(n-1)} f\left(U_{*}(\sigma)\right)\right] d \sigma
$$

we have

$$
\begin{aligned}
U_{*}(s) & =\int_{s_{K}^{*}}^{s} U_{*}^{\prime}\left(s^{\prime}\right) d s^{\prime} \\
& =-(n-1)^{2} \int_{s_{K}^{*}}^{s} \int_{s^{\prime}}^{\infty}\left[h_{B}\left(r, U_{*}(\sigma)\right)-\sigma^{-2 n /(n-1)} f\left(U_{*}(\sigma)\right)\right] d \sigma d s^{\prime} .
\end{aligned}
$$

We need to show that the last integrand is absolutely integrable on the region $\left\{\left(\sigma, s^{\prime}\right): \sigma>s^{\prime}>s_{K}^{*}\right\}$. The term $h_{B}$ offers no problem, since $\left|h_{B}\right|=O\left(\sigma^{-2 n /(n-1)}\right)$. 
To handle the second term we first observe that $\left|U_{*}(\sigma)\right| \leq A+A \sigma^{\epsilon}$, where $\epsilon$ may be picked arbitrarily small and $A=A(\epsilon)$. Since $\left|f\left(U_{*}\right)\right| \leq \mu_{N}\left|U_{*}\right|$, we see that

$$
\left|\sigma^{-2 n /(n-1)} f\left(U_{*}(\sigma)\right)\right|=O\left(\sigma^{\epsilon-2 n /(n-1)}\right),
$$

so that picking $\epsilon<2 /(n-1)$ shows the second term in the integrand is absolutely integrable. We have

$$
\begin{aligned}
0 & >u_{*}(0)=U_{*}(\infty) \\
& =-(n-1)^{-2} \int_{s_{K}^{*}}^{\infty}\left(\sigma-s_{K}^{*}\right)\left[h_{B}\left(\sigma, U_{*}(\sigma)\right)-\sigma^{-2 n /(n-1)} f\left(U_{*}(\sigma)\right)\right] d \sigma>-\infty .
\end{aligned}
$$

The proof of the case $n=1$ proceeds very similarly.

Next consider the case $n>1$, where the values of $B, \underline{c}$ and the sign of $u^{\prime}(1)$ are chosen so that the solution (1) with slope $\pm \underline{c} \sqrt{B f(B)}$ at $r=1$ has at least $K+1$ roots on $(1 / 2,1)$ and $u(r)<0$ on $\left(r_{K}, r_{K-1}\right)$. We will denote this solution by $u_{\underline{c}}$. We will also consider the solutions $v_{M}$ to

$$
\begin{gathered}
\left(r^{n} v_{M}^{\prime}\right)^{\prime}=r^{n}\left[f(B) \phi-\psi\left(r, v_{M}\right)-f\left(v_{M}\right)\right], \\
v_{M}(0)=M, \quad v_{M}^{\prime}(0)=0,
\end{gathered}
$$

and pick $B$ so large that the leftmost negative minimum is less than $-D$ and occurs on $[0,1 / 2)$ (see Lemma 14 and Remark 7). Let $\underline{M}$ denote the smallest value of $M$ such that the leftmost local minimum of $v_{M^{\prime}}$ is less than $-D$ for all $M^{\prime}>M$. For $M>\underline{M}$ let the two leftmost roots of $v_{M}+D$ be denoted by $l(M)$ and $\rho(M)$, where $0<l(M)<\rho(M)$. Next, pick $\bar{M}$ so large that $\rho(\bar{M})-l(\bar{M}) \geq 1$. That this is possible follows from Lemma 8(i) and the fact that the location of the root $l(M)$ will tend to zero while the slope at the first root will increase indefinitely in magnitude as $M$ increases (by Lemma 13). Note that $\rho(M)-l(M) \rightarrow 0$ as $M \rightarrow \underline{M}$, so that we may define $\rho(\underline{M})=l(\underline{M})$ by continuity. Define

$$
\mu=\inf _{\underline{M} \leq M \leq \bar{M}} \rho(M) .
$$

Of course, $\mu>0$. Next we consider the solutions $u_{c}$ to (1) with slope $u_{c}^{\prime}(1)=$ $(c / \underline{c}) u_{\underline{c}}^{\prime}(1)$. Once $\underline{c}$ and $B$ have been picked we may increase $B$ further so as to ensure that the local minimum of $u_{c}, c \geq \underline{c}$, that lies between $r_{K}$ and $r_{K-1}$ is less than $-D$. To see this, we first note that increasing $B$ can be accomplished without decreasing the number of roots between $1 / 2$ and 1 . This follows from Lemma 9(i) and equation (18). Since, for $c \geq \underline{c}, u_{c}$ will attain a minimum value less than $-D$ between $r_{K}$ and $r_{K-1}$, there must be unique values $L(c)$ and $R(c)$, $r_{K}<L(c)<R(c)<r_{K-1}$, where $u_{c}(L(c))=u_{c}(R(c))=-D$. Moreover, there exists a value $\bar{c}$, sufficiently large so that $L(\bar{c})<\mu$. To see this, we use the first half of the proof. As $c$ increases, it must approach a value $c_{*}$ at which the root $r_{K}$ is lost. On compact sets in $(0,1]$ the solution will approach $u_{*}$, the solution with $u_{*}^{\prime}(0)=0$ and $u_{*}(0)$ negative.

COMPLETE ARGUMENT THAT $s_{K} \rightarrow 0$ as $c \rightarrow \infty$.

Since $u_{c} \rightarrow u_{*}$ uniformly on compact sets in $(0,1]$, it is necessary for $L(c) \rightarrow 0$ as $c \uparrow c_{*}$. Hence, there must indeed exist a value $\bar{c}$ such that $L(\bar{c})<\mu / 2$. Finally, let

$$
\Phi: \mathcal{Q}=:[\underline{c}, \bar{c}] \times[\underline{M}, \bar{M}] \rightarrow R^{2}
$$


be the map defined by

$$
\Phi(c, M)=(l(M)-L(c), \rho(M)-l(M)+L(c)-R(c)) .
$$

We easily see that $\Phi(c, M)$ points outward on $\partial \mathcal{Q}$, and therefore, by virtue of the homotopy invariance of the topological degree, we have

$$
\operatorname{deg}(\Phi, \mathcal{Q}, 0)=\operatorname{deg}(\mathrm{id}, \mathcal{Q}, 0)=1,
$$

so that $\Phi$ attains the value $(0,0)$ someplace in $\mathcal{Q}$. That is to say, there exist values $c_{0}, M_{0}$ such that both $v_{M_{0}}$ and $u_{c_{0}}$ satisfy equation (1) with boundary conditions $u_{c_{0}}\left(\alpha_{0}\right)=v_{M_{0}}\left(\alpha_{0}\right)=-D, u_{c_{0}}\left(\beta_{0}\right)=v_{M_{0}}\left(\beta_{0}\right)=-D$, where $\alpha_{0}=L\left(c_{0}\right)=l\left(M_{0}\right)$ and $\beta_{0}=R\left(c_{0}\right)=\rho\left(M_{0}\right)$. Also both $u_{c_{0}}$ and $v_{M_{0}}$ are less than $-D$ on $\left(\alpha_{0}, \beta_{0}\right)$, so we may apply Lemma 8 (ii) to conclude that $u_{c_{0}} \equiv v_{M_{0}}$. This means we have proved the first two parts of the following theorem.

Theorem 2. Suppose $H_{1}-H_{4}$ are satisfied and $K$ is any nonnegative integer. There exists a constant $B_{K}$ such that whenever $B \geq B_{K}$, then

(i) For each integer $0 \leq k \leq K$ equation $(1)+(2)$ has a solution $u_{k}^{(-)}$with $u_{k}^{(-)}(0)<0$, and having precisely $k$ roots in $(0,1)$.

(ii) For each integer $1 \leq k \leq K$ problem $(1)+(2)$ has a solution $u_{k}^{(+)}$with $u_{k}^{(+)}(0)>0$, and having precisely $k$ roots in $(0,1)$.

(iii) For sufficiently large $B$ there is no positive solution $u$ for problem $(1)+(2)$ with $u^{\prime}(0)=0, u(1)=0$.

Proof. We only need to prove (iii). To accomplish this, we need a more careful estimate for positive solutions than that obtained in Lemma 5(iii). In particular, we need to improve the estimate (8). Let $u$ be a positive solution of (1) with $u(1)=0, u^{\prime}(1) \leq 0$. Let $\beta_{*}$ be the largest values in $(0,1)$ where $u(r)=B_{*}$. We have $\alpha<\alpha_{*}<\beta_{*}<1$. On $\left[\beta_{*}, 1\right]$ we have (we will only prove the case $n>1$ )

$$
\begin{aligned}
\left(u^{\prime} r^{n}\right)\left(u^{\prime} r^{n}\right)^{\prime} & =[f(B) \phi-\psi-f(u)] r^{2 n} u^{\prime} \\
& \leq\left[f\left(B_{*}\right)-f(u)\right] \beta_{*}^{2 n} u^{\prime} .
\end{aligned}
$$

Integrating from $r$ to 1 , we have

$$
u^{\prime}(r)^{2} \geq 2\left(\frac{\beta_{*}}{r}\right)^{2 n}\left[f\left(B_{*}\right) u(r)-F(u(r))\right]+u^{\prime}(1)^{2} .
$$

Since $F(u) / u$ is increasing, we have $F(u) / u \leq F\left(B_{*}\right) / B_{*}$ if $0 \leq u \leq B_{*}$, and hence

$$
u^{\prime}(r) \leq-\sqrt{2}\left(\frac{\beta_{*}}{r}\right)^{n}\left[f\left(B_{*}\right)-F\left(B_{*}\right) / B_{*}\right]^{1 / 2} u(r)^{1 / 2} .
$$

Integrating once again, this time from $\beta_{*}$ to 1 , we obtain

$$
-2 B_{*}^{1 / 2} \leq \sqrt{2}\left[f\left(B_{*}\right)-F\left(B_{*}\right) / B_{*}\right]^{1 / 2} \beta_{*}\left(\beta_{*}^{n-1}-1\right) /(n-1)
$$

or

$$
\beta_{*}^{2}\left(1-\beta_{*}^{n-1}\right)^{2} \leq \frac{2 B_{*}(n-1)^{2}}{f\left(B_{*}\right)-F\left(B_{*}\right) / B_{*}} .
$$

As $B \rightarrow \infty$ we have $B_{*} \rightarrow \infty$, so that the right-hand side tends to zero. In view of (29) we cannot have $\beta_{*} \rightarrow 0$, since that would not be compatible with $u^{\prime}(r)$ tending 
to infinity at $u^{-1}(\sigma)$ for each $\sigma \in(0, B)$. Hence $\beta_{*} \rightarrow 1$. Moreover, by (30) we see that

$$
\left|u^{\prime}\left(\beta_{*}\right)\right| \geq \sqrt{2}\left[B_{*} f\left(B_{*}\right)-F\left(B_{*}\right)\right]^{1 / 2} \rightarrow \infty
$$

as $B_{*} \rightarrow \infty$ by Lemma 2(iii). Indeed, since $u-F(u) / f(u)$ is increasing on $(0, \infty)$ we see that

$$
\left|u^{\prime}\left(\beta_{*}\right)\right| \geq C f\left(B_{*}\right)
$$

for some constant $C$ and sufficiently large $B_{*}$. By Lemma 5 (iii) applied to $u-B_{*}$ we see that $\beta_{*}-\alpha_{*} \rightarrow 0$ as $B_{*} \rightarrow \infty$. It remains to show that $\alpha_{*}-\alpha \rightarrow 0$ as $B_{*} \rightarrow 0$. To see this we proceed as before:

$$
r^{n} u^{\prime}\left(r^{n} u^{\prime}\right)^{\prime} \leq[f(B)-f(u)] \alpha_{*}^{2 n} u^{\prime} .
$$

Integrating from $r$ to $\alpha_{*}$, we have

$$
\frac{1}{2} \alpha_{*}^{2 n} u^{\prime}\left(\alpha_{*}\right)^{2}-\frac{1}{2} r^{2 n} u^{\prime}(r)^{2} \leq \alpha_{*}^{2 n}\left[f(B) B_{*}-F\left(B_{*}\right)+F(u(r))-f(B) u(r)\right]
$$

or

$$
\begin{aligned}
u^{\prime}(r)^{2} & \geq\left(\frac{\alpha_{*}}{r}\right)^{2 n} u^{\prime}\left(\alpha_{*}\right)^{2}-2\left(\frac{\alpha_{*}}{r}\right)^{2 n}\left[f(B) B_{*}-F\left(B_{*}\right)+F(u(r))-f(B) u(r)\right] \\
& \geq\left(\frac{\alpha_{*}}{r}\right)^{2 n}\left[C^{2} f\left(B_{*}\right)^{2}-2 f(B) B_{*}\right] .
\end{aligned}
$$

But $\lim _{B \rightarrow \infty}\left[C^{2} f\left(B_{*}\right)^{2}-2 f(B) B_{*}\right]=\infty$. Taking the square root of (32) and integrating, we have

$$
\begin{aligned}
\alpha^{1-n}-\alpha^{* 1-n} & \leq \frac{(n-1) B_{*}}{\alpha_{*}^{n}\left[C^{2} f\left(B_{*}\right)^{2}-2 f(B) B_{*}\right]^{1 / 2}} \\
\alpha^{*}-\alpha & \leq\left(\frac{\alpha}{\alpha_{*}}\right)\left[B_{*} / f\left(B_{*}\right)\right]\left[C^{2}-2 \frac{f(B)}{f\left(B_{*}\right)} \frac{B_{*}}{f\left(B_{*}\right)}\right]^{-1 / 2} \longrightarrow 0 \quad \text { as } \quad B \rightarrow \infty .
\end{aligned}
$$

\section{REFERENCES}

1. A. Ambrosetti and G. Prodi, On the inversion of some differentiable mappings with singularities between Banach spaces, Ann. Math. Pura Appl. 93 (1972), 231-246. MR 47:9377

2. A. Castro and S. Gadam, The Lazer McKenna conjecture for radial solutions in the $R^{N}$ ball, Elec. J. Diff. Eq., 1993, No. 7, 1-6. MR 94j:35049

3. A. Castro and A. Kurepa, Radially symmetric solutions to a superlinear Dirichlet problem in a ball with jumping nonlinearities, Trans. Amer. Math. Soc. 315 (1989), 353-372. MR 90g:35053

4. E. Hille, Lectures on Ordinary Differential Equations, Addison-Wesley, Reading, MA, 1969. MR 40:2939

5. A. C. Lazer and P. J. McKenna, Large amplitude periodic oscillations in suspension bridges: some new connections with nonlinear analysis, SIAM Review 32 (1990), 537-578. MR 92g:73059

6. S.J. Pohožaev, Eigenfunctions of the equation $\Delta u+\lambda f(u)=0$, Soviet Math. Doklady 6 (1965), 1408-1411. MR 33:411

Department of Mathematics, University of North Texas, Denton, Texas 76203

Department of Mathematics, Arizona State University, Tempe, Arizona 85287-1804 